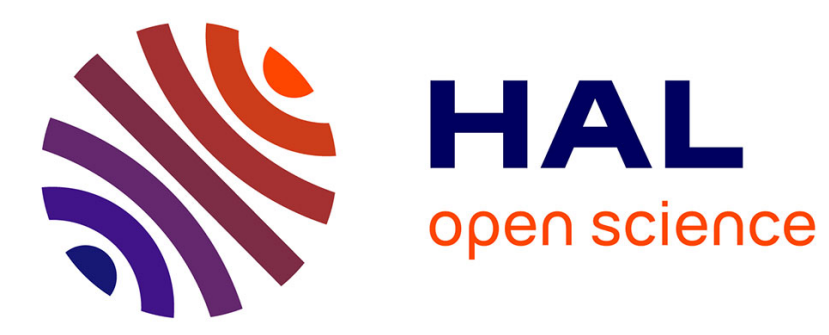

\title{
Grenoble Traffic Lab: An experimental platform for advanced traffic monitoring and forecasting
}

Carlos Canudas de Wit, Fabio Morbidi, Luis Leon Ojeda, Alain Kibangou, Iker Bellicot, Pascal Bellemain

\section{- To cite this version:}

Carlos Canudas de Wit, Fabio Morbidi, Luis Leon Ojeda, Alain Kibangou, Iker Bellicot, et al.. Grenoble Traffic Lab: An experimental platform for advanced traffic monitoring and forecasting. IEEE Control Systems, 2015, 35 (3), pp.23-39. 10.1109/MCS.2015.2406657 . hal-01059126v3

\section{HAL Id: hal-01059126 \\ https://hal.science/hal-01059126v3}

Submitted on 3 Feb 2015

HAL is a multi-disciplinary open access archive for the deposit and dissemination of scientific research documents, whether they are published or not. The documents may come from teaching and research institutions in France or abroad, or from public or private research centers.
L'archive ouverte pluridisciplinaire HAL, est destinée au dépôt et à la diffusion de documents scientifiques de niveau recherche, publiés ou non, émanant des établissements d'enseignement et de recherche français ou étrangers, des laboratoires publics ou privés.

$$
\text { Copyright }
$$




\section{Grenoble Traffic Lab}

\section{An experimental platform for advanced traffic monitoring and forecasting}

Carlos Canudas de Wit, Fabio Morbidi, Luis León Ojeda, Alain Y. Kibangou,

Iker Bellicot, Pascal Bellemain

"The start from the Ocean House was something marvelous to see. The drivers stormed and scolded, the women shrieked and cried, wheels locked at intervals of perhaps ten minutes. Occasionally, too, a carriage would capsize, and be hauled over to the fence for repairs ... [It was] like a huge funeral procession, crawling along at a snail's pace. It was a feat to get to the city at all." This is the report of newspaper The Examiner of what happened when a multitude of attendants and their carriages turned to leave at the same time after the end of a horse race at Ingleside Race Track near San Francisco, California, on November 16, 1873, probably one of the oldest traffic jams on record. Nowadays, motor traffic jams in road networks occur regularly and have a critical impact on modern cities in terms of productivity loss, air pollution and wasteful energy consumption [1]. According to the INRIX Traffic Scorecard Report [2], in 2013 French drivers wasted, on average, 35 hours in traffic, and France tied for third place with Germany in Europe, in terms of traffic jams (after Belgium and the Netherlands). The situation is not better in the United States, where the top three worst traffic cities in 2013 were Los Angeles, Honolulu and San Francisco where drivers have spent 64, 60 and 56 hours in traffic jams, respectively. In order to address the traffic issue, since the '80s intelligent transportation systems (ITS) have emerged to enhance the infrastructure efficiency and provide congestion relief. ITS applications, such as 
dynamic route guidance with variable message panels, highway access control and travel-time forecasting, are now being employed successfully worldwide.

Several technologies are available today for collecting traffic data: stationary detectors such as Doppler radars, single and double inductive-loop detectors, laser, infrared sensors, magnetometers and video cameras, are now routinely used in the field, and they have being gradually supplemented by a growing amount of data obtained from mobile detectors or tracing vehicles: this includes continuous tracers (floating car data, FCD, i.e. satellite geolocalization, and floating mobile data, FMD, i.e. localization via the mobile-phone network), and point-to-point tracers (Bluetooth tags from telephones and onboard radios, radio-frequency identification for electronic toll collection, WiFi positioning system, WPS, i.e. localization from WiFi hotspots) [3]-[5]. Data from stationary detectors, also known as "cross-sectional data", complements, in several respects, that coming from mobile detectors: in fact, while stationary sensors provide a better temporal coverage of traffic, continuous tracers are able to produce highly-accurate trajectories for single vehicles. However, the former are typically more expensive to install but easier to operate in the long term. The problems of fusing data from heterogeneous sources and of data assimilation have become increasingly important in recent years, and are the subject of active research, see [5, Ch. 5.3] and [6]. Data assimilation is the process by which observations are incorporated into a model of a real system. It is a cyclic procedure: in each cycle, measurements of the current (and possibly past) state of a system are combined with the results from a model (the forecast) to produce an analysis, which is considered as the "the best" estimate of the current state of the system. The model is then advanced in time and its result becomes the forecast in the next analysis cycle [5]. A major breakthrough in highway traffic modeling came from the discovery of a relationship between traffic density and flow at a certain location, through the fundamental diagram. This diagram is at the basis of the first 
fluid-dynamic macroscopic model proposed by Lighthill, Whitham and Richards in the '50s, the LWR model, for details, see "Fluid-Dynamic Macroscopic Models for Highway Traffic". More recently, the cell transmission model (CTM) [7] and the related switching mode model (SMM) [8] have attracted considerable attention in the transportation and control literatures. The SMM is a piecewise-affine state-dependent discrete-time system based on the CTM which is well suited for model-based traffic estimation [8]-[12] and control [13]-[15]: in fact, it represents a good compromise between mathematical simplicity and modeling accuracy (see the second case study below).

In spite of the aforementioned technological and theoretical advances, the mathematical physics community, which has been developing increasingly-sophisticated dynamical traffic models, and the traffic engineering community, which is more concerned with the collection, statistical analysis and interpretation of real traffic data, have not been able to establish durable links and a common language so far. In particular, despite the numerous ITS initiatives worldwide, to the best of the authors' knowledge there do not exist, at present, easy-to-use experimental platforms which allow to test and compare the performance of advanced traffic management and monitoring algorithms on highway data. In order to fill this gap and provide a standardized testbed for the validation of new theoretical work, the NeCS team, a joint CNRS (GIPSA-lab) and Inria team-project, has recently developed the Grenoble traffic lab (GTL). A source of inspiration for GTL was Caltrans Performance Measurement System (PeMS) and Tools for Operational Planning (TOPL) [16], [17]. GTL is an innovative platform for online collection of traffic data coming from a dense wireless sensor network (130 magnetometers over $10.5 \mathrm{~km}$ ) installed in the south ring of the city of Grenoble in France, "Rocade sud" in French. It is worth pointing out here that differently from a sophisticated and general-purpose system such as PeMS (which can be virtually operated in any road-network topology, directly imported from Google Maps), GTL works on a 


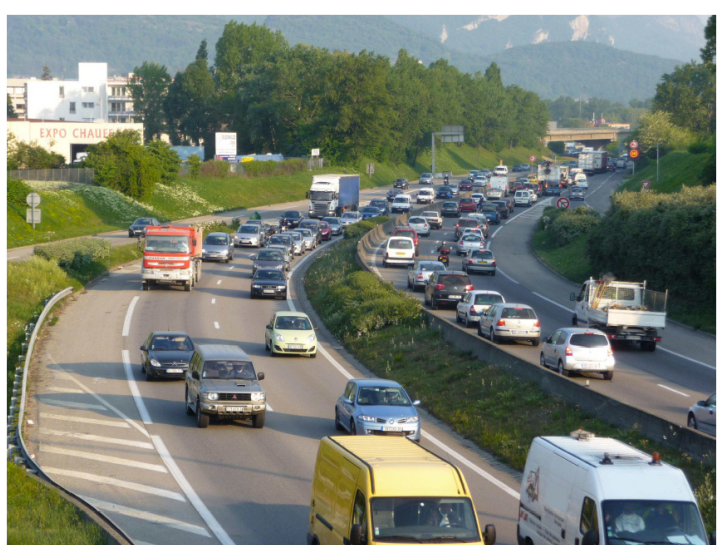

(a)

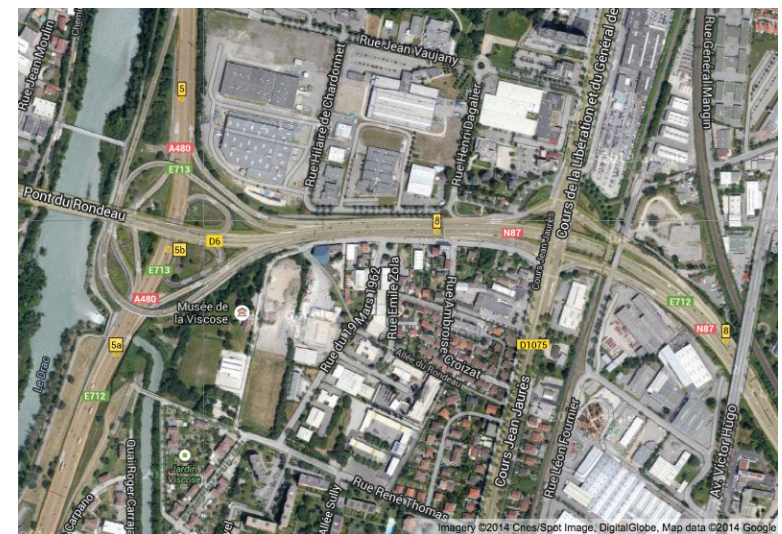

(b)

Figure 1. The south ring of Grenoble. (a) View of a stretch of the south ring, direction north-east towards Meylan (Image courtesy of DIR-CE); (b) Aerial view of the interchange "Rondeau" at the west end of the south ring (left center in the image): this site endures heavy traffic congestion during the morning and afternoon rush hours (Image courtesy of Google Maps/Satellite).

smaller scale and fully covers a single peri-urban corridor: however, this specificity constitutes also one of its distinctive strengths. GTL is the culmination of a four-year research effort and it became operative in winter 2013: the full potential of the system was demonstrated during a Show Day on traffic modeling, estimation and control, organized at Grenoble in May 2014. The synergy with the local traffic authorities which endorsed the large-scale deployment of a novel sensor technology, and the characteristic daily congestion experienced at the interchange "Rondeau" (see Fig. 1), make the south ring of Grenoble well-suited for traffic studies and an attractive working environment for students, practitioners and researchers in the control, applied mathematics, and transportation communities.

The goal of this paper is to present the main functional components of GTL, with a special emphasis on traffic estimation and forecasting problems. Space constraints forced us to omit extensive research on ramp-metering control in the south ring, conducted with state-of-the-art 
traffic simulators [18]: for more details, the reader is referred to [19], [20]. In what follows, we will proceed to describe in more detail the site of interest and the architecture of GTL. After presenting the results of statistical analyses on the magnetometer data, we will focus on two relevant control systems applications, and conclude the article by highlighting some promising directions for future research.

\section{Site of Interest and GTL Architecture}

Grenoble covers an area of $18.13 \mathrm{~km}^{2}$ and with a total population of 159953 (as of January 2014, source INSEE) is the 16th largest city in France. The city is relatively flat with an average elevation of 221 meters. The surface circulation is made difficult by the presence of mountains enclosing the city in the north, west, and south-east sides, and by the confluence of the Isère and Drac rivers in the north-west side in the direction of Lyon. These natural boundaries have prevented the construction of a highway surrounding the city, thus making vehicle circulation problematic especially during the peak hours: Grenoble was the third most congested city in France in 2013, with 41 hours wasted on average by the drivers in traffic [2]. The south ring of Grenoble, "route nationale 87", is a highway enclosing the southern part of the city from A41 to A480, completed in 1985. It consists of two carriageways with two lanes, it has 10 on-ramps and 7 off-ramps in the internal roadway, and it stretches between the satellite city of Meylan $\left(45.20531^{\circ} \mathrm{N}, 5.78353^{\circ} \mathrm{E}\right)$, and the interchange Rondeau $\left(45.15864^{\circ} \mathrm{N}\right.$, $5.70384^{\circ} \mathrm{E}$ ), for an overall length of about $10.5 \mathrm{~km}$, see Fig. 2. The south ring is a crucial transportation corridor for Grenoble. The total usage of both directions is about 90000 vehicles per day, with peaks of up to 110000 . About $5 \%$ of the traffic is trucks. The highway is operated by the Direction Interdépartementale des Routes Centre-Est (DIR-CE) and the speed limit ranges between $70 \mathrm{~km} / \mathrm{h}$ and $90 \mathrm{~km} / \mathrm{h}$. Because of its higher criticality for vehicular circulation and 


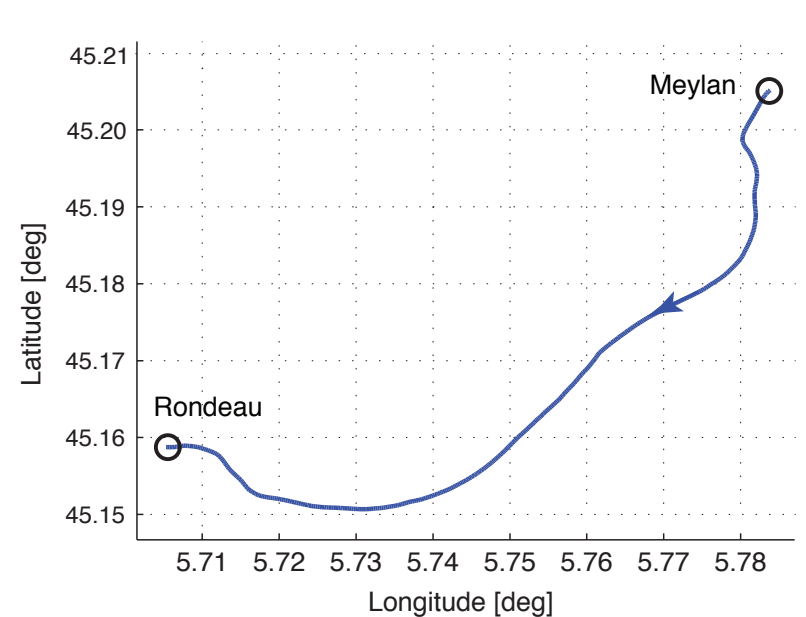

(a)

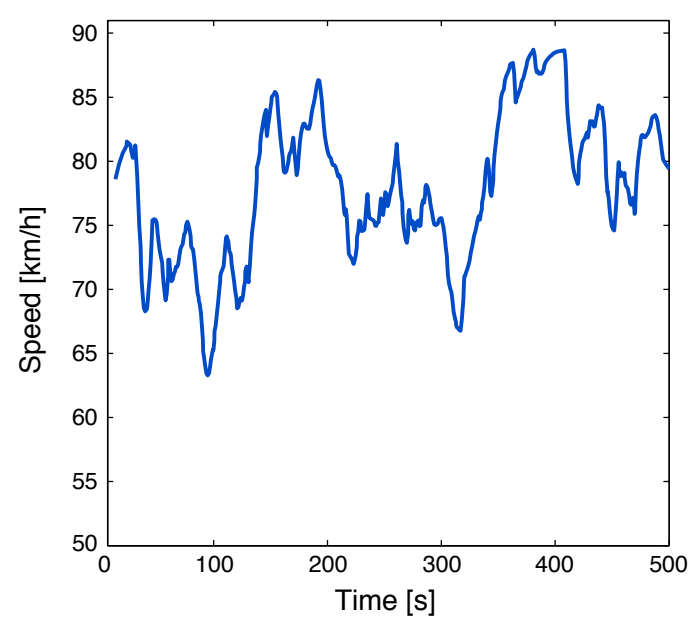

(c)
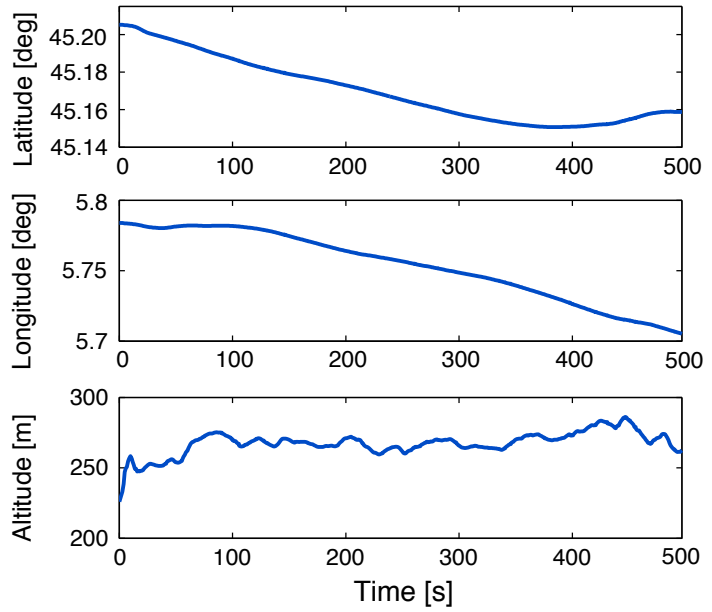

(b)

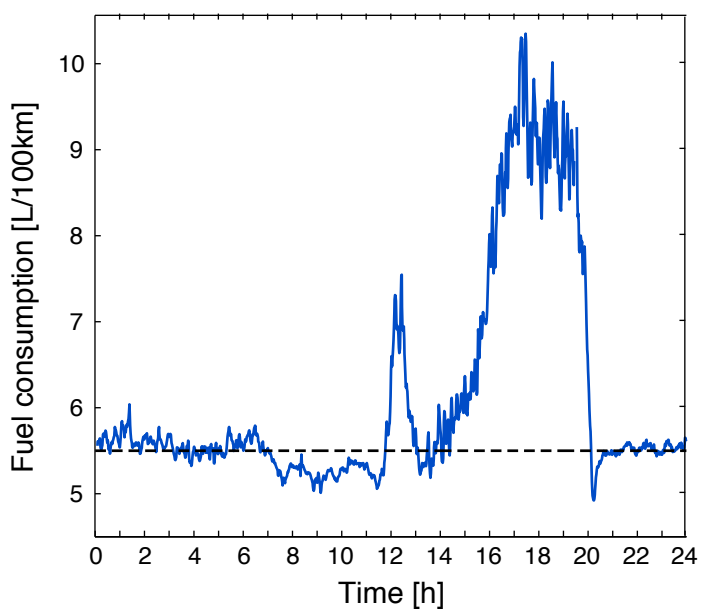

(d)

Figure 2. Traveling the south ring of Grenoble. (a) Spatial trajectory, and (b), (c) time evolution of the position and speed of a car in the south ring on Thursday, December 5, 2013 between 19:48,00 and 19:56,26, recorded with the GPS-based smartphone application "My Tracks"; (d) Time history of the fuel consumption of an average diesel-powered family car in conditions of heavy congestion on February 28, 2014, estimated using a physics-based modal model (the black dashed line indicates the "nominal" consumption under light traffic, cf. [5, Sect. 20.4]). 
budget limitations of the NeCS team, in GTL only the east-west direction of the south ring of Grenoble, the carriageway on the left in Fig. 1(a), is considered. In light-traffic conditions to travel from Meylan to Rondeau takes about 7 minutes and 30 seconds (see Fig. 2(a)-(c)), while under heavy congestion the travel time can grow up to 45 minutes and fuel consumption up to $80 \%$ (cf. Fig. 2(d)).

In the remainder of this section, we will describe the different functional levels of GTL. The reader is referred to Fig. 3 for a workflow diagram of GTL architecture.

\section{Level 1: Physical Layer}

The south ring has been equipped with 54 pairs of Sensys Networks VDS240 3-axis wireless magneto-resistive sensors embedded in the middle of the fast/slow lanes 4.5 meters apart, plus 20 sensors in the on-/off-ramps (cf. Fig. 4 and Fig. 5, and Table I), for details, see "How Do Magnetic Sensors Detect a Passing Vehicle?". The magnetometers have a sampling rate of $128 \mathrm{~Hz}$ and are powered with non-rechargeable primary lithium thionyl chloride ( $\left.\mathrm{Li}-\mathrm{SOCl}_{2}\right)$ 3.6 V, 7.2 Ah batteries which guarantee 10 years of operation and up to 300 million vehicle detections. The magnetometers provide macroscopic information, such as flow $\phi[\mathrm{veh} / \mathrm{h}]$, timemean speed $v[\mathrm{~km} / \mathrm{h}]$ and occupancy [\%] (the fraction of time during which the cross-section is occupied by a vehicle) as well as microscopic information, such as single-vehicle speed, inter-vehicle time gap and vehicle length. The latter piece of information can be used, for example, for safety or vehicle-class distribution analyses: however, for the sake of simplicity, in the rest of this article we will exclusively deal with macroscopic data. Notice that since $\phi=\rho v$, the density $\rho[\mathrm{veh} / \mathrm{km}]$ can be estimated from the available flow and speed measurements. The magnetometers use an ultra-low power 2.4 GHz TDMA protocol to communicate with a nearby access point (configured and remotely operated with Sensys software "TrafficDOT2"), which sends the data to a server in the Grenoble traffic control center at the DIR-CE via 


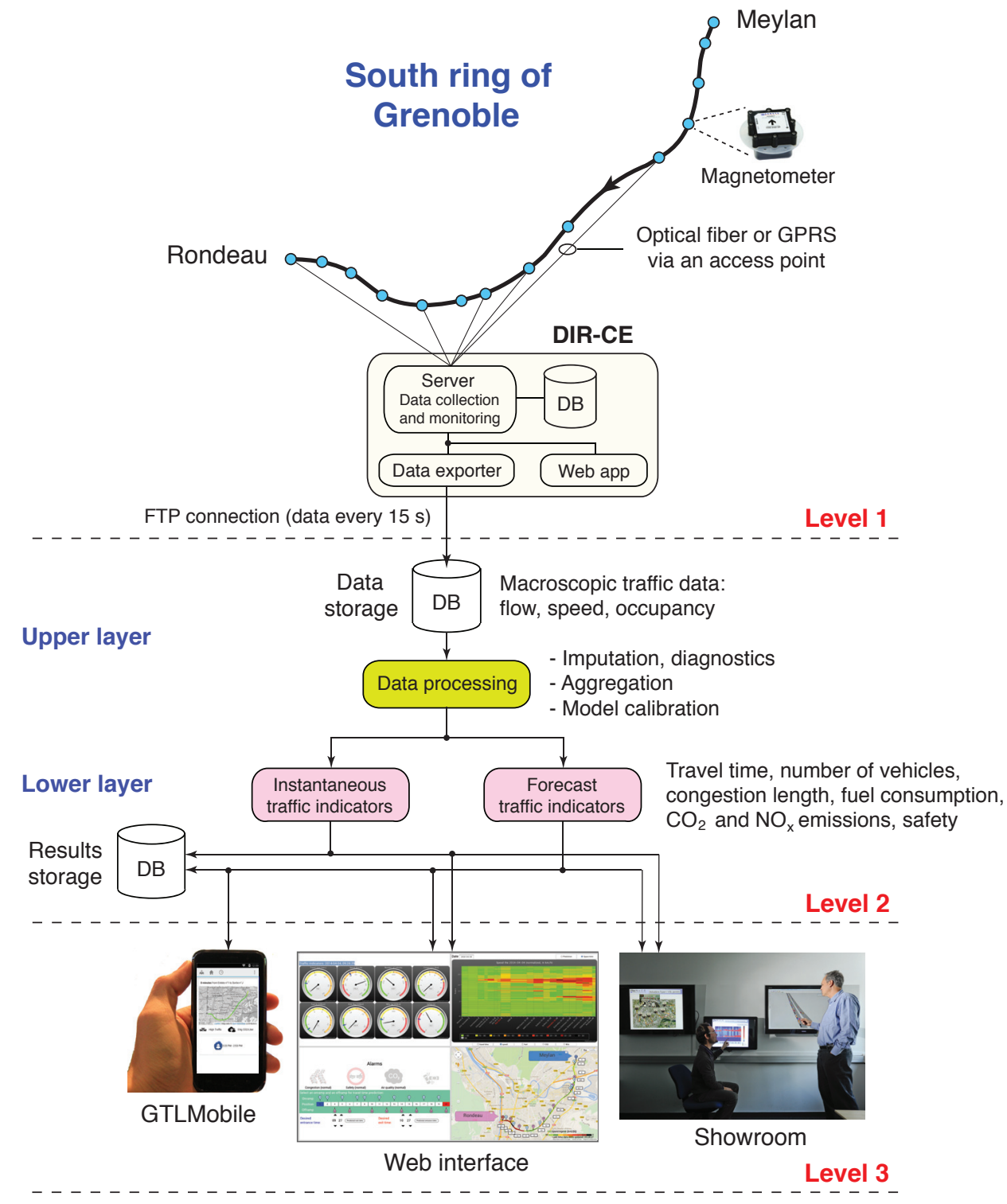

Figure 3. Three-level architecture of GTL. Level 1: physical layer, Level 2: data processing and applications, Level 3: results display.

optical fiber, "f”, or via a wireless GPRS (General Packet Radio Service) connection, "g”, see Table I. If the magnetometer, which has a transmit/receive bit rate of $250 \mathrm{kbps}$, is outside a radius of 45 meters from the access point, a repeater (mounted on the vertical signage) is used to relay the signal to it. A total of 19 access points and 21 repeaters are active in the 


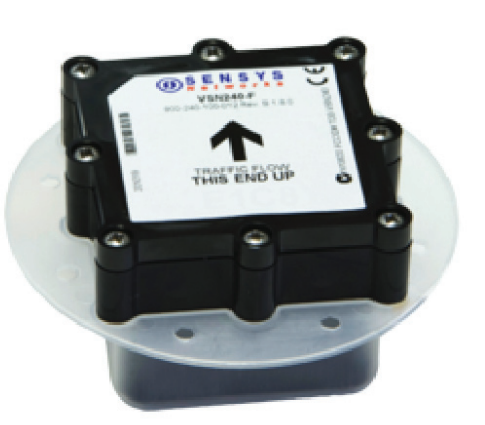

(a)

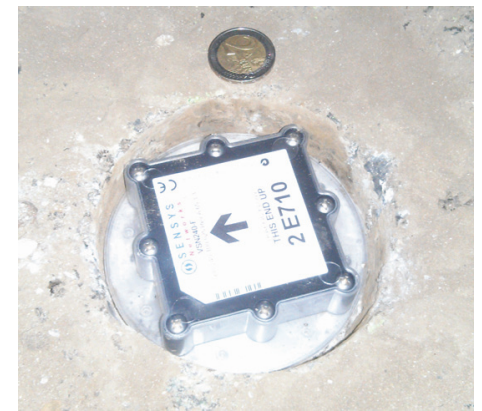

(b)

Figure 4. Magnetic sensors. (a) Sensys Networks VDS240 protected by a hardened plastic case (With permission of Sensys Networks, Inc.); (b) A magnetometer in its final location in the south ring, about $3 \mathrm{~cm}$ below the road surface, before being covered with fast-drying epoxy (the arrow points in the direction of traffic flow). A 2 Euro coin is shown near the sensor for comparison: the actual size is $7.4 \mathrm{~cm} \times 7.4 \mathrm{~cm} \times 4.9 \mathrm{~cm}$ and the weight is 300 grams.

south ring. The traffic data are monitored and stored in a database (DB in short) at DIR-CE, where every 15 seconds an FTP data exporter pushes them to a server located at Inria Grenoble Rhône-Alpes, see Fig. 3. The access to the raw traffic data is currently restricted to the members of our research group. The physical installation and configuration of the magnetometers, and testing of the platform took approximately one year, between September 2012 and September 2013: the overall sensor network became fully operational at the end of 2013. A time-consuming statistical analysis of the speed profiles of the magnetometers was necessary to validate location and labeling (note that the sensors sharing the same communication channel have the same hexadecimal serial number or ID, see Table I). Calibration issues related to the vehicle-counting task and problems of data consistency over time were also encountered, cf. the section "Platform Operation and Data Validation" below. 


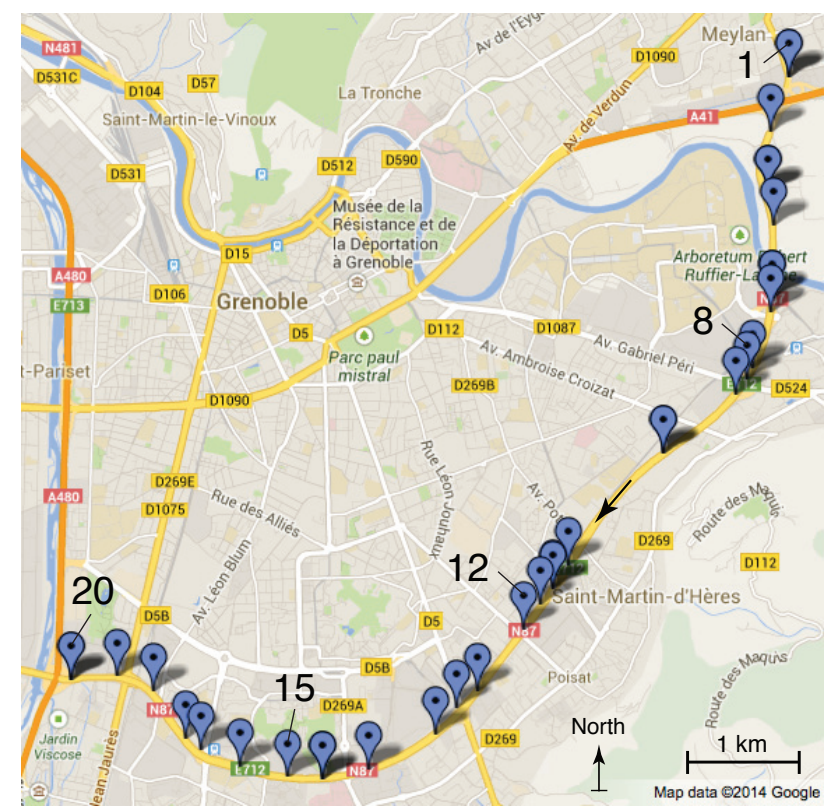

(a)
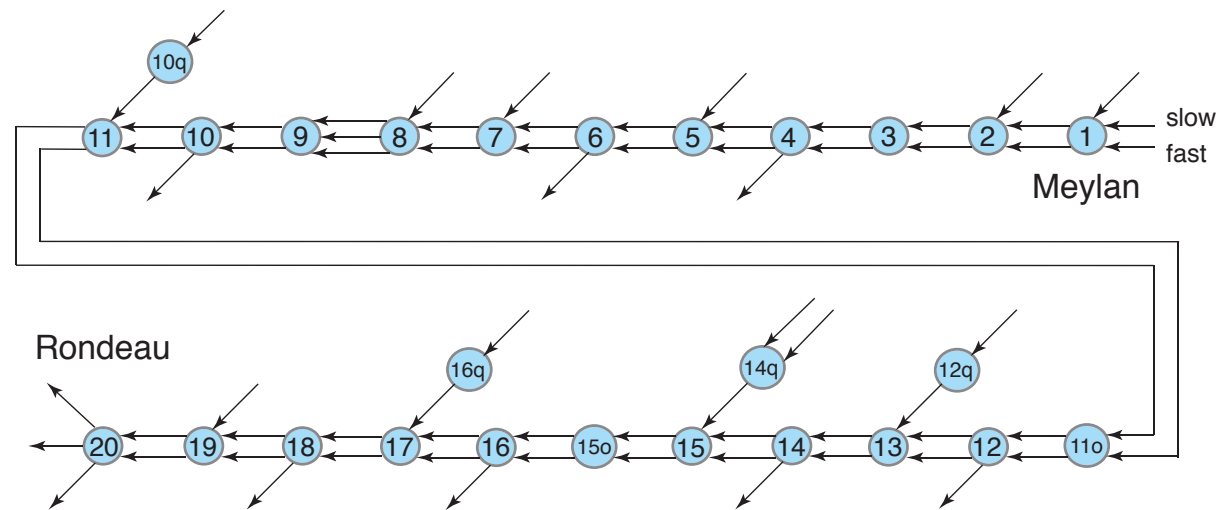

(b)

Figure 5. Sensor disposition in the south ring. (a) Location of the collection points, blue flags (Image courtesy of Google Maps); (b) Graphical representation of road interconnections: the cyan disks correspond to the collection points and the arrows to the lanes (fast, slow, on-ramp, off-ramp, etc.) equipped with magnetometers, see Table I.

\section{Level 2: Data Processing and Applications}

Level 2 consists of an upper and lower layer, which are here described in detail. 


\begin{tabular}{|c|l|c|c|c|}
\hline $\mathrm{n}^{\circ}$ & Name & Lanes & ID, Comm. & Position [km] \\
\hline \hline 1 & Meylan & Slow, Fast, On-ramp & $3356, \mathrm{f}$ & 0.000 \\
\hline 2 & A41 Grenoble & Slow, Fast, On-ramp & $3354, \mathrm{f}$ & 0.405 \\
\hline 3 & Taillat (or Carronnerie) & Slow, Fast & $343 \mathrm{c}, \mathrm{f}$ & 1.168 \\
\hline 4 & Domaine Univ. (exit) & Slow, Fast, Off-ramp & $343 \mathrm{~b}, \mathrm{f}$ & 1.770 \\
\hline 5 & Domaine Univ. (entrance) & Slow, Fast, On-ramp & $343 \mathrm{~b}, \mathrm{f}$ & 1.946 \\
\hline 6 & Gabriel Péri (exit) & Slow, Fast, Off-ramp & $3445, \mathrm{f}$ & 2.470 \\
\hline 7 & Gabriel Péri (entrance 1) & Slow, Fast, On-ramp & $3445, \mathrm{f}$ & 2.604 \\
\hline 8 & Gabriel Péri (entrance 2) & Slow, Middle, Fast, On-ramp & $1 \mathrm{~b} 67, \mathrm{~g}$ & 2.803 \\
\hline 9 & SMH & Slow, Fast & $3357, \mathrm{f}$ & 3.619 \\
\hline 10 & SMH Centre (exit) & Slow, Fast, Off-ramp & $0 \mathrm{ddd}, \mathrm{f}$ & 4.881 \\
\hline $10 \mathrm{q}$ & SMH Centre (queue) & On-ramp & $0 \mathrm{ddd}, \mathrm{g}$ & 5.093 \\
\hline 11 & SMH Centre (entrance) & Slow, Fast, On-ramp & $3355, \mathrm{f}$ & 5.406 \\
\hline $11 \mathrm{o}$ & SMH Centre (overequip.) & Slow, Fast & $3355, \mathrm{f}$ & 5.606 \\
\hline 12 & Eybens (exit) & Slow, Fast, Off-ramp & $21 \mathrm{~d} 1, \mathrm{f}$ & 6.291 \\
\hline $12 \mathrm{q}$ & Eybens (queue) & On-ramp & $21 \mathrm{~d} 1, \mathrm{f}$ & 6.507 \\
\hline 13 & Eybens (entrance) & Slow, Fast, On-ramp & $343 \mathrm{f}, \mathrm{f}$ & 6.770 \\
\hline 14 & Échirolles (exit) & Slow, Fast, Off-ramp & $1 \mathrm{~b} 5 \mathrm{c}, \mathrm{g}$ & 7.418 \\
\hline $14 \mathrm{q}$ & Échirolles (queue) & On-ramp left, On-ramp right & $1 \mathrm{~b} 5 \mathrm{c}, \mathrm{g}$ & 7.742 \\
\hline 15 & Échirolles (entrance) & Slow, Fast, On-ramp & $25 \mathrm{eb}, \mathrm{f}$ & 7.981 \\
\hline $15 \mathrm{o}$ & Échirolles (overequip.) & Slow, Fast & $25 \mathrm{eb}, \mathrm{f}$ & 8.243 \\
\hline 16 & États Généraux (exit) & Slow, Fast, Off-ramp & $25 \mathrm{ea}, \mathrm{f}$ & 8.637 \\
\hline $16 \mathrm{q}$ & États Généraux (queue) & On-ramp & $1 \mathrm{c} 99, \mathrm{~g}$ & 9.015 \\
\hline 17 & États Généraux (entrance) & Slow, Fast, On-ramp & $13 \mathrm{c6}, \mathrm{f}$ & 9.195 \\
\hline 18 & Libération (exit) & Slow, Fast, Off-ramp & $3444, \mathrm{f}$ & 9.645 \\
\hline 19 & Libération (entrance) & Slow, Fast, On-ramp & $25 \mathrm{ec,} \mathrm{f}$ & 10.049 \\
\hline 20 & Rondeau & Left, Middle, Right & $343 \mathrm{e}, \mathrm{f}$ & 10.346 \\
\hline
\end{tabular}

TABLE I

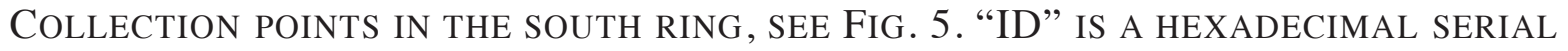
NUMBER ASSOCIATED TO GROUPS OF MAGNETOMETERS. THE COMMUNICATION IS VIA OPTICAL FIBER, "f”, OR GPRS, “g”.

- Upper layer: the raw macroscopic traffic data coming every 15 seconds from the Sensys magnetometers (see Level 1) are stored in a database and then passed through a suite of signal-processing algorithms (green box in Fig. 3), which perform: 
- Imputation and diagnostics: if some data are lost or erroneous (for instance, as a result of communication problems or temporary sensor malfunction), suitable imputation algorithms [21], [22] are run for filling in the missing data with estimated values, see Level 3. In this respect, each magnetometer is evaluated not as a standalone but together with its neighbors and their past measurement history (see [23] and "Vehicle counting" under "Data analysis" in [24]).

- Aggregation: high-resolution traffic data tend to be noisy. In order not to capture dynamics that are not physically meaningful, it is then fundamental to aggregate the data into time slots of 1,5 or 6 minutes, depending on the scenario under investigation. Even after the aggregation of the raw traffic data, high-frequency oscillations might still be present because of data-collection latency and intrinsic measurement noise: it may be then opportune to apply a low-pass filter with an appropriate cut-off frequency (e.g. a first-order low-pass Butterworth filter).

- Model calibration: if model-based algorithms are utilized in the lower layer for computing the traffic indicators (see below), the parameters of the (fluid-dynamic) models are automatically estimated from the data: for example, a least-squares based method inspired by [25] is employed for computing the parameters of the fundamental diagram in the SMM, which is in turn used for traffic-density estimation.

- Lower layer: in this layer the pre-processed data is utilized to compute, at the present time and in the future, several traffic indicators: the travel time [min], the number of vehicles, the congestion length $[\mathrm{km}]$, the fuel consumption $[\mathrm{L} / 100 \mathrm{~km}]$, and $\mathrm{CO}_{2}[\mathrm{~kg} / 100 \mathrm{~km}], \mathrm{NO}_{\mathrm{x}}$ [g/100km] emissions for an average family car and the safety index [s] (see the magenta boxes in Fig. 3). The fuel consumption and $\mathrm{CO}_{2}$ emissions are estimated using a physicsbased modal consumption model [5, Sect. 20.4] for a diesel-powered vehicle $(61.3 \%$ of 
French private cars were diesel in January 2013, according to the INSEE), while for the $\mathrm{NO}_{\mathrm{x}}$ emissions we relied on the statistical modal model proposed in [26]. Finally, the safety index is computed according to a constant time-headway spacing policy with a nominal time headway of two seconds as a reference [27]. As it is known, the "twosecond distance rule" tells a driver the minimum distance needed to reduce the risk of collision with the vehicle directly in front, under ideal driving conditions: two seconds is a safety buffer, and the rule attempts to respect a distance which is consistent with human reaction time (approximately 1.5-2 s). For the sake of simplicity, the algorithms that generate the aforementioned indicators are coded as Simulink blocks: MEX files are used to interface the blocks with the database on one side and with the result-visualization tools (see Level 3, below) on the other. The MATLAB/Simulink environment offers great flexibility and modularity, and allows the user to easily add or delete connections between the blocks and implement new traffic-management algorithms. The instantaneous and forecast indicators yielded by our Simulink blocks are stored in a dedicated database. More details about two algorithms for traffic density estimation and travel-time forecasting recently developed by the our group, are given in the forthcoming "Case studies" section.

\section{Level 3: Results Display}

The indicators computed by the algorithms in Level 2 can be visualized using different media, including a:

- Web interface: the interface includes four panels, see Fig. 6. In the upper-left panel, eight gauges display the indicators relative to the instantaneous traffic conditions in the south ring (together with the worst daily values: blue pointers). The upper-right panel reports spacetime heat maps relative to the current and forecast traffic indicators, and by clicking on the right top dialog box, predicted time-indexed curves are displayed. In the lower-left panel, 


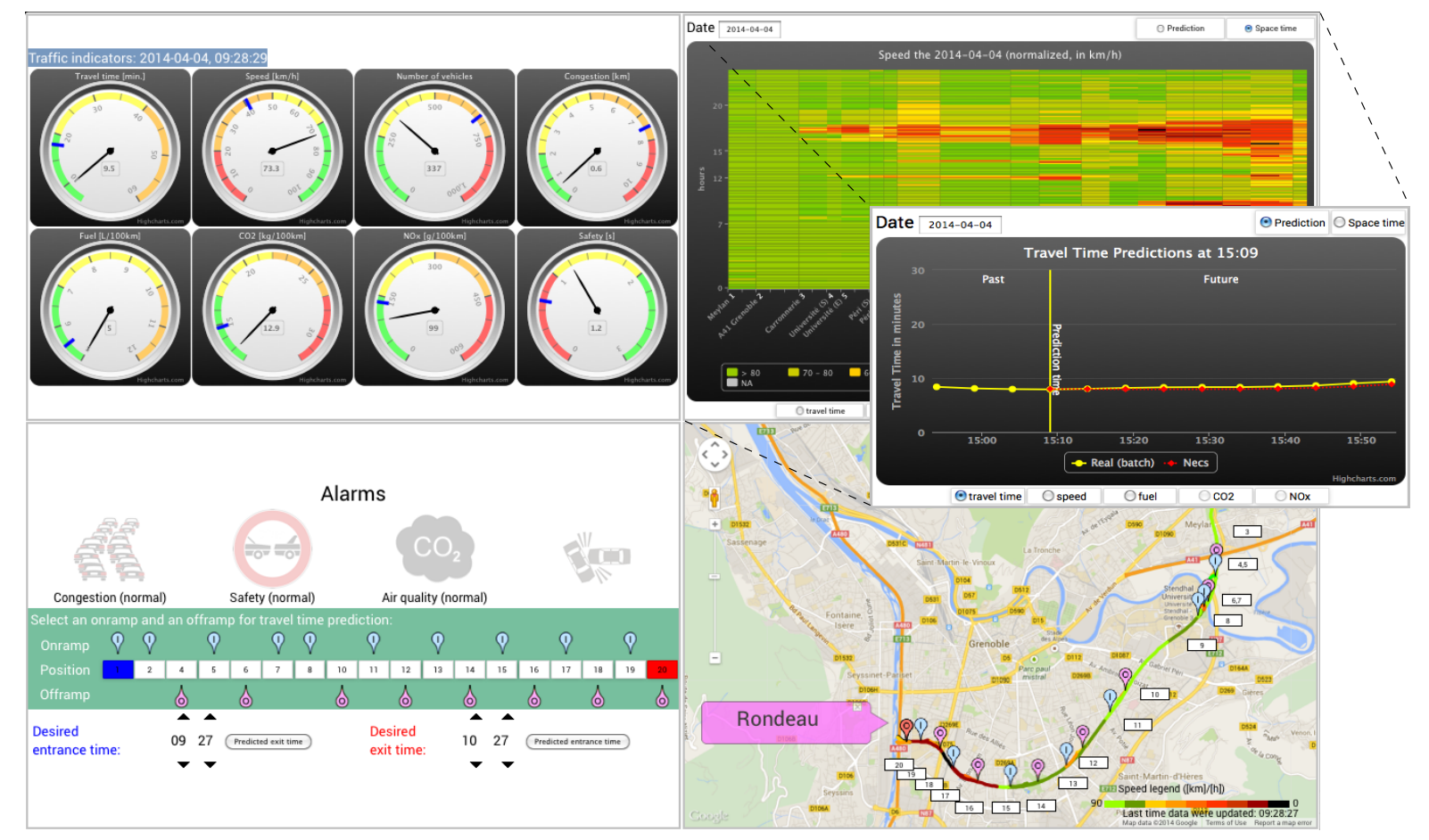

Figure 6. The four panels of GTL web interface. Clockwise from top left: 1) gauges displaying the indicators relative to the instantaneous traffic conditions, 2) space-time heat map relative to the current and forecast traffic indicators, and predicted time-indexed curves, 3) selection of the on-ramp/off-ramp in the south ring and computation of the forecast exit/entrance times, 4) visualization of the collection points in the south ring, and of color-coded average traffic speed in each road segment (Image courtesy of Google Maps).

the user can select an on-ramp and an off-ramp of the south ring and compute the forecast arrival/departure times. Four alarms, in the form of flashing images, are also displayed in this portion of the interface. Finally, the lower-right panel, which has been partially built upon Google Maps, shows the collection points in the south ring, and the color-coded average traffic speed in each road segment. For demonstration purposes, the web interface is available at the address in [28]. 
- Mobile device: an Android smartphone application called "GTLMobile" has been developed in collaboration with the Institut Carnot LSI of Grenoble, to display salient traffic information (forecast travel time and $\mathrm{CO}_{2}$ emissions) to the drivers. The functionalities of the application have been defined by collecting the traveling preferences of over 200 commuters of the south ring via an online questionnaire.

- Showroom: the four panels of the web interface, plus additional diagnostic information about data quality (vehicle-counting performance), are displayed around the clock in seven monitors in a dedicated room at Inria Grenoble Rhône-Alpes.

\section{Platform Operation and Data Validation}

In this section we describe the traffic profiles of a typical weekday in the south ring, and present the results of a statistical data analysis that we conducted to test the performance of the network of magnetometers. These studies were instrumental in developing and setting up the GTL platform.

\section{Analysis of Typical Traffic Patterns}

In order to design effective and reliable traffic estimation and forecasting algorithms, it is crucial to be fully aware of the physical limits of the infrastructure and of recurrent traffic patterns. Fig. 7(a) reports the speed contour of the south ring for the fast and slow lanes on Thursday, January 16, 2014: as it is evident in the figure (horizontal red stripes) heavy congestion originating from the Rondeau interchange, a bottleneck where the speed limit decreases from 90 to $70 \mathrm{~km} / \mathrm{h}$ and the highway branches off south, west and north, is experienced during the morning and afternoon rush hours. In Fig. 7(b), we reported the time evolution (from 2:00 onward) of mainstream flow (black) and speed (green) in location 16 (États Généraux, exit) for 


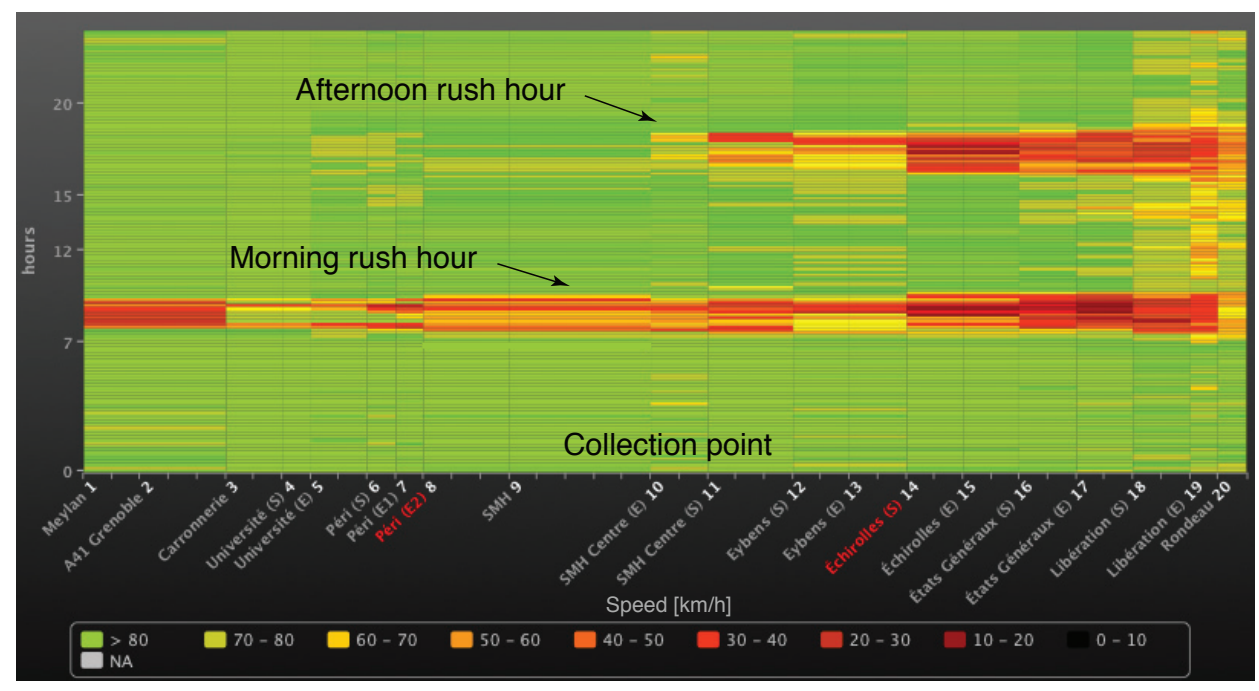

(a)

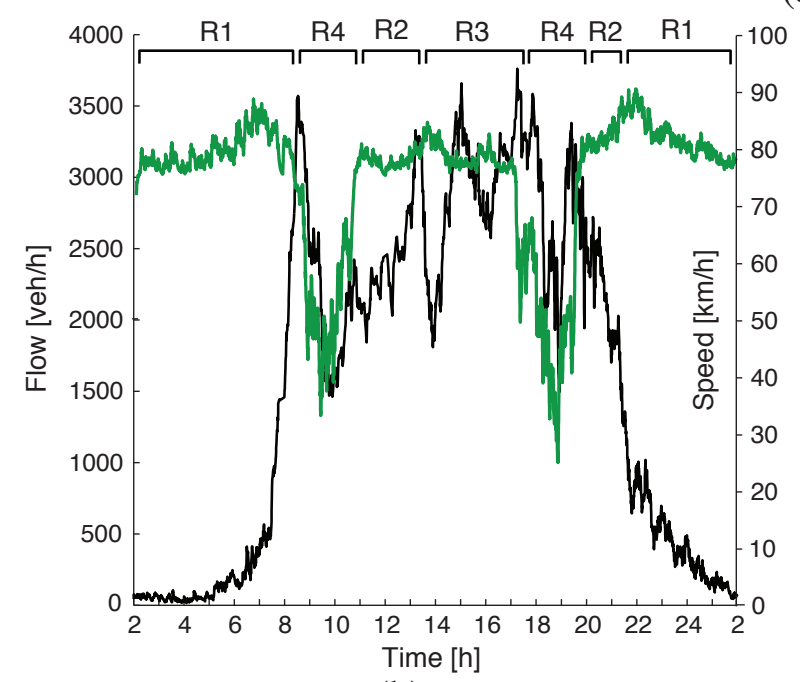

(b)

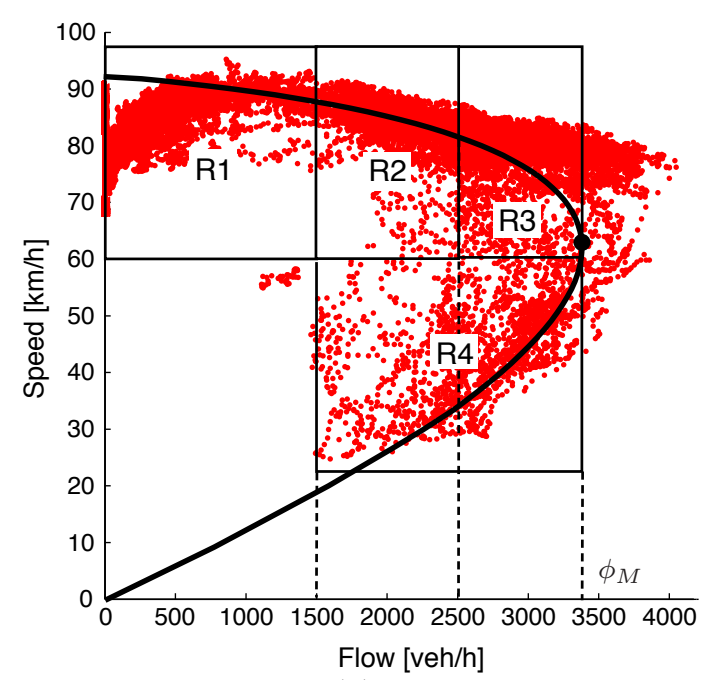

(c)

Figure 7. Typical traffic patterns in the south ring. (a) Speed contour of January 16, 2014: the two red horizontal stripes correspond to the morning and afternoon rush hours; (b) Time evolution of mainstream flow [veh/h] (black) and speed $[\mathrm{km} / \mathrm{h}]$ (green) in location 16 on January 16, 2014; (c) Vehicle speed against traffic flow for five weekdays (January 7, 8, 9, 10 and 16, 2014) at location 16. In (b) and (c), four traffic regimes, R1, R2, R3, R4, are highlighted.

January 16, 2014: to improve the readability, the raw signals have been filtered using a first-order low-pass Butterworth filter. From this figure we can notice that the minimal volume of traffic is at 


\begin{tabular}{|l|c|c|c|}
\hline Traffic regime & Flow [veh/h] (2 lanes) & Aver. speed [km/h] & Approx. time interval \\
\hline \hline R1, Light & $0 \leq \phi<1500$ & $v>60$ & $21: 00-7: 00$ \\
\hline R2, Fluid & $1500 \leq \phi<2500$ & $v>60$ & $9: 30-13: 00,19: 00-21: 00$ \\
\hline R3, Intense & $2500 \leq \phi \leq \phi_{M}$ & $v>60$ & $13: 00-17: 00$ \\
\hline R4, Congested & $1500 \leq \phi \leq \phi_{M}$ & $v \leq 60$ & $7: 00-9: 30,17: 00-19: 00$ \\
\hline
\end{tabular}

TABLE II

THE FOUR TRAFFIC REGIMES IN THE SOUTH RING.

3:00 in the morning and that four traffic regimes, $\mathrm{R} 1, \mathrm{R} 2, \mathrm{R} 3, \mathrm{R} 4$, can be identified in a typical working day (see Table II): regime R1 corresponds to light traffic conditions (early morning and late evening), R3 to the highway operating near the maximal capacity $\phi_{M}$, and R4 is relative to the morning and afternoon traffic peaks, where speed drops below $40 \mathrm{~km} / \mathrm{h}$. Finally, in the intermediate regime R2, the traffic load is moderate (see also "Speed calendar" under "Data analysis" in [24]). The four regimes are also displayed in Fig. 7(c), where we plotted vehicle speed against traffic flow (red dots) for five weekdays, January 7, 8, 9, 10 and 16, 2014, at location 16. The black curve in the figure has been obtained via least-squares fitting using an (implicit) exponential function of flow and speed, $F(\phi, v)=a \exp \left(-b\left(\frac{\phi-c}{v-d}\right)^{a}\right)$, where $a$ (even), $b, c, d$ are positive parameters to be determined. The tip of this curve approximately represents the maximal capacity of the highway at location 16 , i.e. the maximal number of vehicles that can cross this location in one hour.

\section{Magnetometers Versus Inductive-Loop Detectors: Performance Comparison}

In order to assess the performance of the magnetic sensors, we compared the flow/speed measurements of Sensys magnetometers with the corresponding measurements of two SIREDO inductive double-loop detectors which belong to a nation-wide traffic-monitoring network [29]. 
These two detectors, which are not part of the GTL platform, are located between collection points 9 and 10, and $11 \mathrm{o}$ and 12, at $4.319 \mathrm{~km}$ and $5.9 \mathrm{~km}$ from Meylan, respectively. They cover the fast and slow lanes, and provide independent flow, speed and occupancy measurements, with an aggregation time of 6 minutes. In our tests, we considered the days between September 2 and September 20, 2013, weekends excluded. During the working hours (8:00-18:00), we observed a good matching between the average-flow (Figs. 8(a), (c)) and average-speed measurements over the 15 days (Figs. 8(b), (d)): however, the data from the loop detectors appeared to be, overall, more correlated and smoother. These findings are consistent with previously published studies [30]-[32].

\section{Flow-Error Analysis}

An additional test was performed to assess the counting performance of Sensys magnetometers in the south ring. In order to make the presentation of the results precise, it is convenient to introduce here some terminology regarding network flow theory [33]. A network $\mathcal{G}$ is a triple consisting of two sets $\mathcal{E}$ and $\mathcal{N}$, the set of arcs and of nodes, respectively, and a function that assigns to each $j \in \mathcal{E}$ a pair $\left(i_{1}, i_{2}\right) \in \mathcal{N} \times \mathcal{N}$ such that $i_{1} \neq i_{2}$. Node $i_{1}$ is called the initial node of $j$ and $i_{2}$ the terminal node. The arc $j$ is said to be incident to $i_{1}$ and $i_{2}$, where these nodes, by virtue of the existence of such an arc, are said to be adjacent to each other. We henceforth assume that $|\mathcal{N}|=n$ and $|\mathcal{E}|=m$, where $|\cdot|$ denotes the cardinality of a set. The node-arc incidence matrix $\mathbf{E}=\left[e_{i j}\right]$ of $\mathcal{G}$ is defined as follows: $e_{i j}=1$ if $i$ is the initial node of the $\operatorname{arc} j, e_{i j}=-1$ if $i$ is the terminal node of the arc $j$, and $e_{i j}=0$ otherwise. Note that $\mathbf{E}$ is an $n \times m$ matrix, and that each column of $\mathbf{E}$ has exactly one +1 and one -1 . The flow of an arc is a variable that measures the quantity of material flowing through an arc of the network. Mathematically, the flow of an arc $j$ is a real nonnegative number which we denote by $\phi_{j}$. In order to analyze what happens to a flow at a certain node $i$, in particular if the node "leaks", 


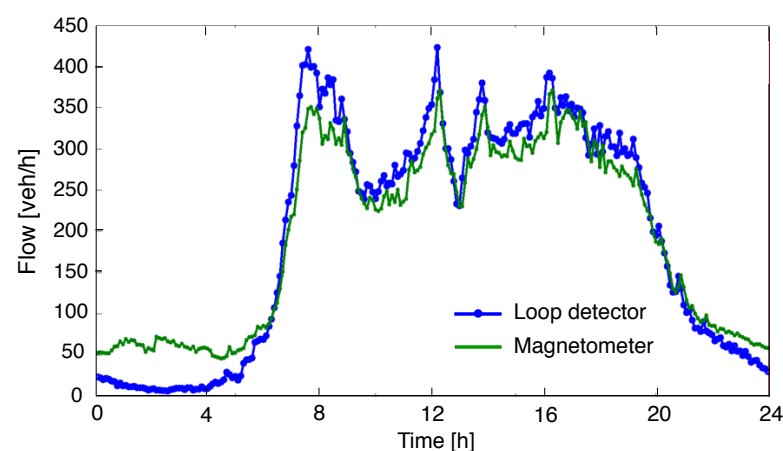

(a)

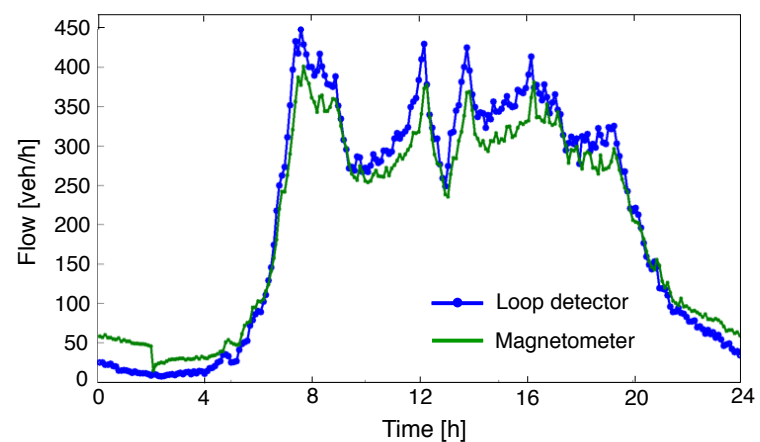

(c)

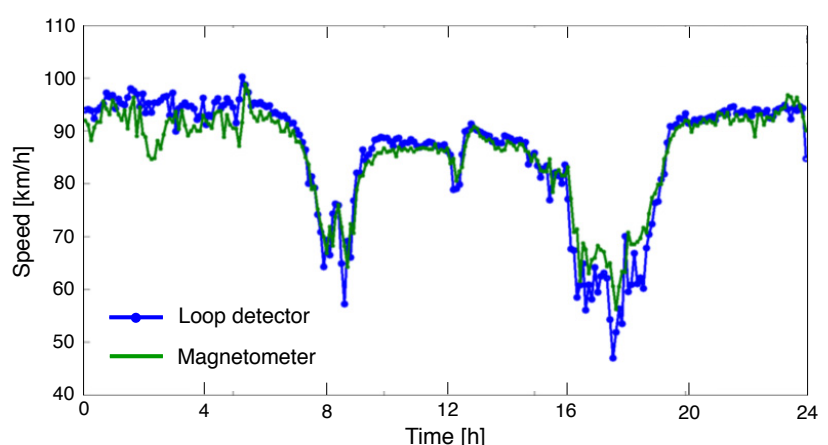

(b)

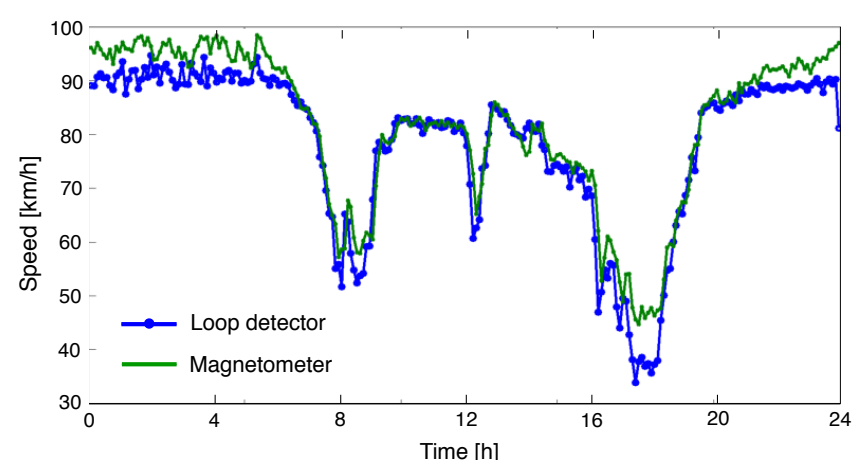

(d)

Figure 8. Magnetometers versus inductive-loop detectors. Comparison between the measurements of magnetometers (green) and inductive-loop detectors (blue) between September 2 and 20, 2013, without weekends. (a), (c) Average flows over the 15 days in correspondence to the first and second loop detector; (b), (d) Average speeds over the 15 days in correspondence to the first and second loop detector.

it is useful to introduce the notion of divergence. Given a network $\mathcal{G}$, the divergence of the flow at node $i \in \mathcal{N}$, denoted by $y_{i}$, is the quantity $y_{i}=\sum_{j \in \mathcal{E}} e_{i j} \phi_{j}$, that is, the total flow departing from node $i$ minus the total flow arriving at $i$. Let $\phi=\left[\phi_{1}, \ldots, \phi_{m}\right]^{T}$ be the flow vector. A node $i$ is said to be a source for the flow vector $\phi$ if $y_{i}>0$ and a sink if $y_{i}<0$. If $y_{i}=0$, the flow is conserved at $i$. Note that if we call $\mathbf{y}=\left[y_{1}, \ldots, y_{n}\right]^{T}$ the divergence vector associated with the flow vector $\phi$, we have that $\mathbf{y}=\mathbf{E} \phi$. In a network, the total amount of flow created at the sources always equals the total amount destroyed at the sinks. This is expressed by the 


\begin{tabular}{|c|c|c|l|c|c|c|c|c|c|c|}
\hline Location & Feb. 7 & Feb. 8 & $\cdots$ & Feb. 15 & Feb. 16 & Feb. 17 & Aver. & Std & Min & Max \\
\hline \hline $1 \rightarrow 2$ & n.a. & n.a. & $\cdots$ & n.a. & n.a. & n.a. & n.a. & n.a. & n.a. & n.a. \\
\hline $2 \rightarrow 3$ & 0.1 & 0.5 & $\cdots$ & 0.9 & 0.1 & 0.4 & 0.04 & 0.57 & -0.8 & 0.9 \\
\hline $3 \rightarrow 4$ & 0.5 & -0.03 & $\cdots$ & 0.5 & 0.3 & 0.5 & 0.33 & 0.23 & -0.04 & 0.7 \\
\hline $4 \rightarrow 5$ & -0.1 & 0.1 & $\cdots$ & 0.1 & -0.05 & 0.02 & 0.01 & 0.1 & -0.19 & 0.1 \\
\hline $5 \rightarrow 6$ & 0.9 & 0.3 & $\cdots$ & -7.6 & 0.2 & -1.2 & -0.94 & 2.51 & -7.6 & 0.9 \\
\hline $6 \rightarrow 7$ & n.a. & n.a. & $\cdots$ & n.a. & n.a. & n.a. & n.a. & n.a. & n.a. & n.a. \\
\hline $7 \rightarrow 8$ & n.a. & n.a. & $\cdots$ & n.a. & n.a. & n.a. & n.a. & n.a. & n.a. & n.a. \\
\hline $8 \rightarrow 9$ & -0.5 & 0.8 & $\cdots$ & -13.6 & -0.5 & -3.4 & -6.31 & 6.54 & -15.4 & 0.8 \\
\hline $9 \rightarrow 10$ & 0.1 & 0.01 & $\cdots$ & -0.2 & -0.3 & -0.2 & -0.19 & 0.2 & -0.5 & 0.1 \\
\hline $10 \rightarrow 11$ & -0.6 & -0.4 & $\cdots$ & -0.1 & -0.4 & -0.6 & -0.39 & 0.16 & -0.6 & -0.1 \\
\hline $11 \rightarrow 11 \mathrm{o}$ & -2 & -1.4 & $\cdots$ & -1.9 & -1.4 & -0.4 & -1.6 & 0.44 & -2 & -0.4 \\
\hline $11 \mathrm{o} \rightarrow 12$ & 2.9 & 2 & $\cdots$ & 2.4 & 2.1 & 0.8 & 2.14 & 0.52 & 0.8 & 2.9 \\
\hline $12 \rightarrow 13$ & 0.2 & -0.04 & $\cdots$ & 0.1 & 0.2 & 0.1 & 0.01 & 0.23 & -0.5 & 0.2 \\
\hline $13 \rightarrow 14$ & -1.7 & -1.5 & $\cdots$ & 13 & -0.4 & 1 & 4.43 & 6.48 & -1.7 & 13.2 \\
\hline $14 \rightarrow 15$ & -3.1 & -1.8 & $\cdots$ & -14 & -1 & -4.9 & -8.23 & 7.03 & -19 & -1 \\
\hline $15 \rightarrow 15 \mathrm{o}$ & -1.3 & -0.2 & $\cdots$ & 0.02 & -0.04 & -1.3 & -0.75 & 0.53 & -1.3 & 0.02 \\
\hline $15 \mathrm{o} \rightarrow 16$ & 0.4 & 0.2 & $\cdots$ & 0.2 & -0.2 & 0.4 & 0.23 & 0.23 & -0.2 & 0.6 \\
\hline $16 \rightarrow 17$ & 0.1 & 0.7 & $\cdots$ & -11.5 & -1 & -2.1 & -5.23 & 6.1 & -13.7 & 1.7 \\
\hline $17 \rightarrow 18$ & -1 & -0.1 & $\cdots$ & -0.7 & -0.2 & -0.5 & -0.62 & 0.32 & -1.1 & -0.1 \\
\hline $18 \rightarrow 19$ & -15.2 & -19.8 & $\cdots$ & -28 & 1.8 & -6.8 & -9.09 & 10.24 & -28 & 9.5 \\
\hline $19 \rightarrow 20$ & -23 & 16.8 & $\cdots$ & 23.6 & -1.6 & -25.5 & -3.56 & 21.78 & -50.5 & 23.6 \\
\hline
\end{tabular}

TABLE III

STATISTICAL ANALYSIS OF THE RELATIVE DIVERGENCE OF DAILY FLOWS [\%] IN THE SOUTH RING OVER 10 DAYS (FROM FEBRUARY 7 TO FEBRUARY 17, 2014). “n.a.” STANDS FOR “NOT AVAILABLE”.

total divergence principle:

$$
\sum_{i \in \mathcal{N}} y_{i}=0 \quad \text { for } \quad \mathbf{y}=\mathbf{E} \boldsymbol{\phi}
$$

The flows $\phi$ in $\mathcal{G}$ such that $\mathbf{E} \phi=0$, i.e. $\phi$ is conserved at every node, are called circulations (note that analogously, in physics, a vector field with constant zero divergence is called incompressible or solenoidal). The set of all circulations forms a linear subspace of $\mathbb{R}^{m}$, the circulation space $\mathscr{C}=\operatorname{ker}(\mathbf{E})$, where $\operatorname{ker}(\cdot)$ denotes the kernel of a matrix. 
Let us now return to the task of evaluating the counting performance of the magnetometers in the south ring of Grenoble. Consider the graphical representation of the south ring in Fig. 5(b), and assume that the 26 Sensys collection points represent the nodes of a network $\mathcal{G}_{\mathrm{SR}}$ and the arrows the arcs of $\mathcal{G}_{\mathrm{SR}}$ (through which vehicles flow). It is then easy to verify that the incidence matrix $\mathbf{E}$ of $\mathcal{G}_{\mathrm{SR}}$ has 26 rows and 70 columns, from which we can compute the daily divergences in the south ring by using the measured daily flows $\phi_{j}, j \in\{1,2, \ldots, 70\}$. In order to simplify the analysis, let us introduce the relative divergence of the daily flow at location $i$ (in \%)

$$
y_{r, i}^{\%}=100 \times \frac{y_{i}}{\max \left\{\left|\sum_{j \in \mathcal{E}: e_{i j}=-1} e_{i j} \phi_{j}\right|, \sum_{j \in \mathcal{E}: e_{i j}=1} e_{i j} \phi_{j}\right\}} .
$$

Note that according to this definition, $y_{r, i}^{\%}$ is a signed quantity. Unfortunately, we will never have $y_{r, i}^{\%}=0$ in the real world, since a counting error will always be present, due to the technological limitation of sensors or to vehicles stuck in a road section: we can then only hope that $\left|y_{r, i}^{\%}\right|<\gamma$, $\forall i$, where $\gamma$ is a suitable small threshold. From an inspection of Table III in which we reported the average, standard deviation, minimal and maximal relative divergence of the daily flow over 10 days in February 2014 (because of the space constraints, Table III does not show the flow of all days), we can notice that except for two locations in which environmental disturbances and vehicle lane-changing are important sources of uncertainty, the absolute value of average relative divergence is always smaller than $7 \%$, see "Vehicle counting" under "Data analysis" in [24]. The counting performance appeared to significantly depend on the sensor calibration accuracy and, notably, on the sensitivity threshold over the three axes of the magnetometers.

\section{Case studies}

GTL provides direct access to real traffic data, which allows, in turn, to synthesize and test new algorithms with unprecedented rapidity and accuracy. In this section we report two examples of recently-developed algorithms which use tools of control systems theory: the first one relies on the adaptive Kalman filter and speed measurements for short-term multi-step ahead 
travel-time forecasting [34], and the second one leverages a Luenberger-like observer based on the CTM for traffic-density estimation [9] in the south ring. The development of these algorithms has greatly benefited from the unique features of the GTL platform.

\section{Multi-Step Ahead Travel-Time Forecasting}

The problem of highway travel-time forecasting has been widely studied and several solutions have been proposed in the literature depending on how the available historical and current-time traffic information is handled [35]-[38]. In this section, we briefly present the main features of a novel travel-time forecasting algorithm based on the noise-adaptive Kalman filter (AKF) (for further details, see [23], [34]). Our algorithm considers progressive traffic conditions, i.e. it accounts for the spatial and temporal conditions encountered by a potential driver along the road, for details, see "Travel Time Forecasting". Fig. 9 shows the main functional blocks of the forecasting algorithm. Three typologies of data, obtained from the available speed measurements, feed the algorithm: the historical travel-time information ("Historical data") from all $M=21$ links of the south ring (a link is defined as the stretch between two collection

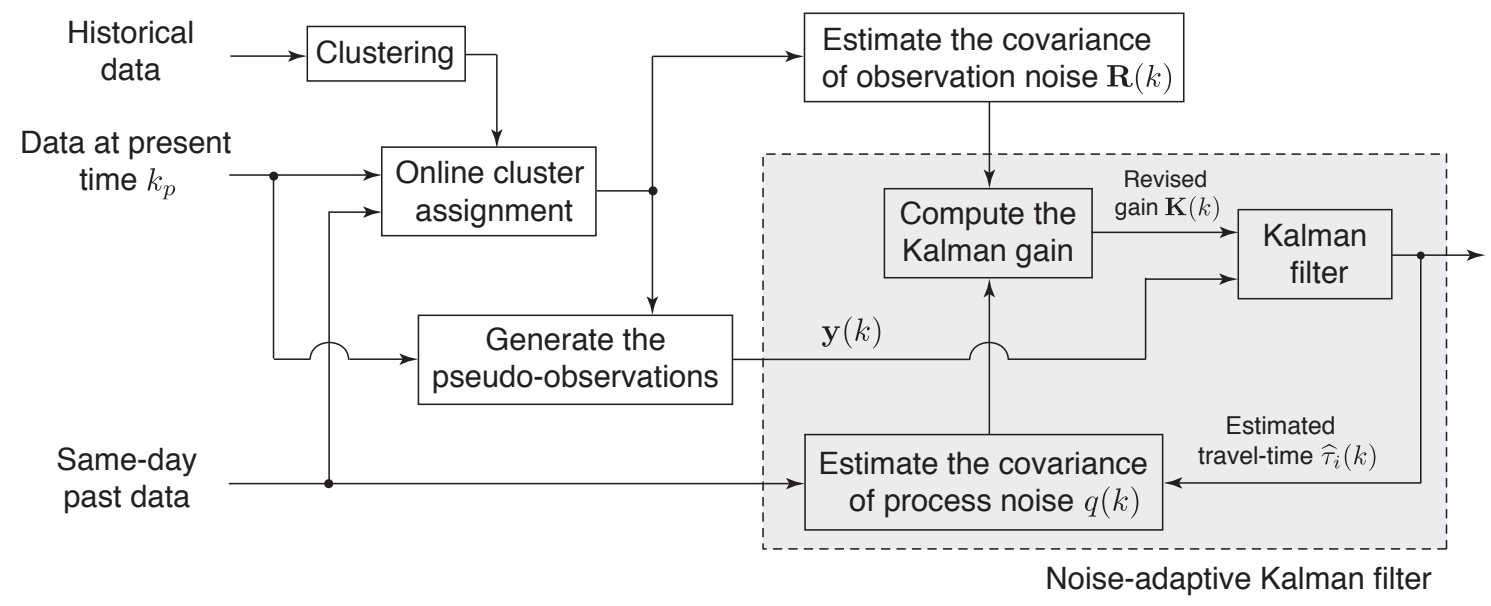

Figure 9. Block diagram of the traffic forecasting algorithm based on the Kalman filter from [23]. 
points, recall Table I), the travel-time information at present time $k_{p}$ in the link $i$, and the travel-time information from midnight of the current day up to time $k_{p}$ in the link $i$ ("Sameday past data"). In order to reduce spatial complexity and maximize correlation, the historical data has been clustered into five time zones (00:00-7:00, 7:00-10:00, 10:00-16:00, 16:00-19:00, and 19:00-24:00) using the standard k-means algorithm (as a preliminary step, a separation into working and non-working days, i.e. Sundays and holidays, is also performed). The clustered data are used to produce pseudo-observations via two predictors which rely on the average of historical data, and on the data at the present time and historical increment, respectively, and to estimate the covariance matrix of the observation noise $\mathbf{R}(k) \in \mathbb{R}^{2 \times 2}$ for $k \in\left\{k_{p}, k_{p}+1, \ldots, k_{p}+H\right\}$, where $H$ is the forecasting horizon. Note that by introducing pseudo-observations the forecasting problem is conveniently converted into a standard filtering problem. Matrix $\mathbf{R}(k)$ and the covariance of the process noise $q(k) \in \mathbb{R}$, which is estimated from the forcing residuals [39, Sect. 4.7], are used to compute a revised gain $\mathbf{K}(k) \in \mathbb{R}^{2 \times 2}$ for the Kalman filter which outputs the estimated travel-time $\widehat{\tau}_{i}(k)$ in link $i$ for $k \in\left\{k_{p}, k_{p}+1, \ldots, k_{p}+H\right\}$. Fig. 10 reports the results of several tests that we conducted under typical traffic conditions. In particular, Figs. 10(a), (c) show the forecast travel time provided by the proposed method (purple solid, circle) against the travel time computed from a simple average of the historical data (blue dashed, cross), and the ground truth (black solid, star) at $k_{p}=8: 45$ on September 17, 2013 and at $k_{p}=17: 15$ on September 11, 2013, respectively. Figs. 10(b), (d) report the corresponding forecast trajectories in the (space, time)-speed plane against the ground truth for departure times at intervals of 15 minutes. From the figures, we notice that the proposed method always outperforms the average of historical data, and that the congestion build-up and phase-out times are correctly captured by the algorithm. For cross-validating the proposed algorithm, let us now introduce the estimated cumulative travel time from the current link $i$ to the destination link $j(i \neq j \in\{1,2, \ldots, M\})$ at 


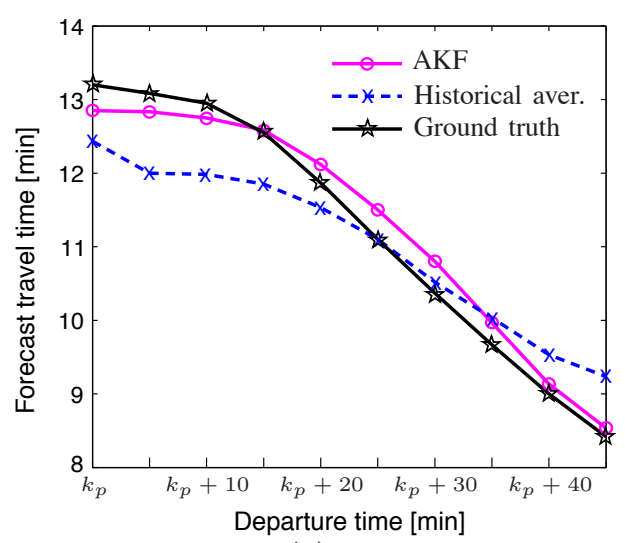

(a)

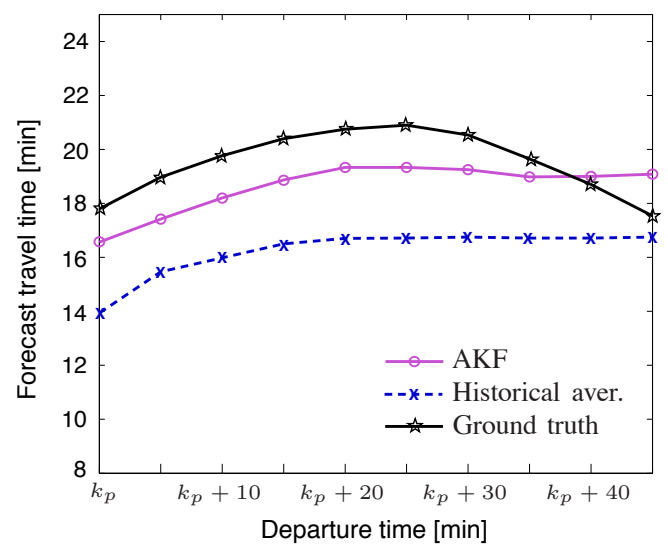

(c)

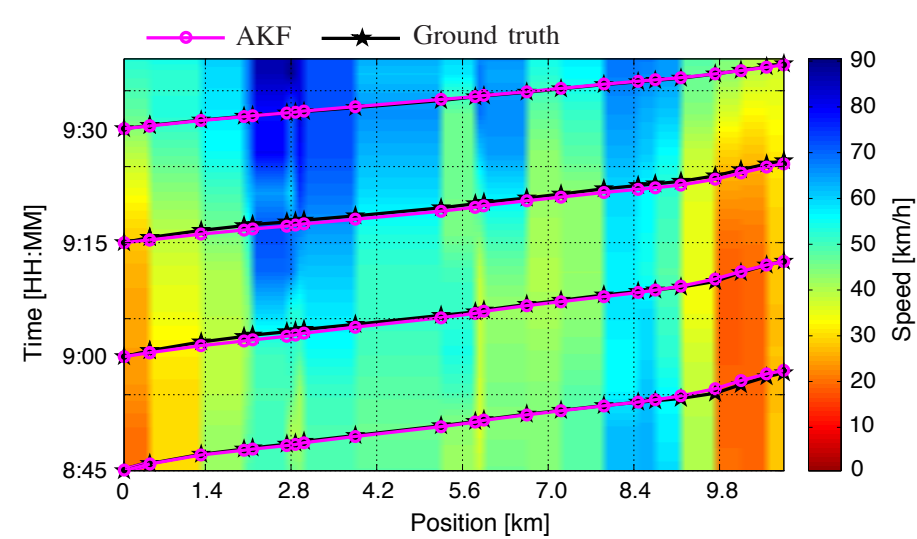

(b)

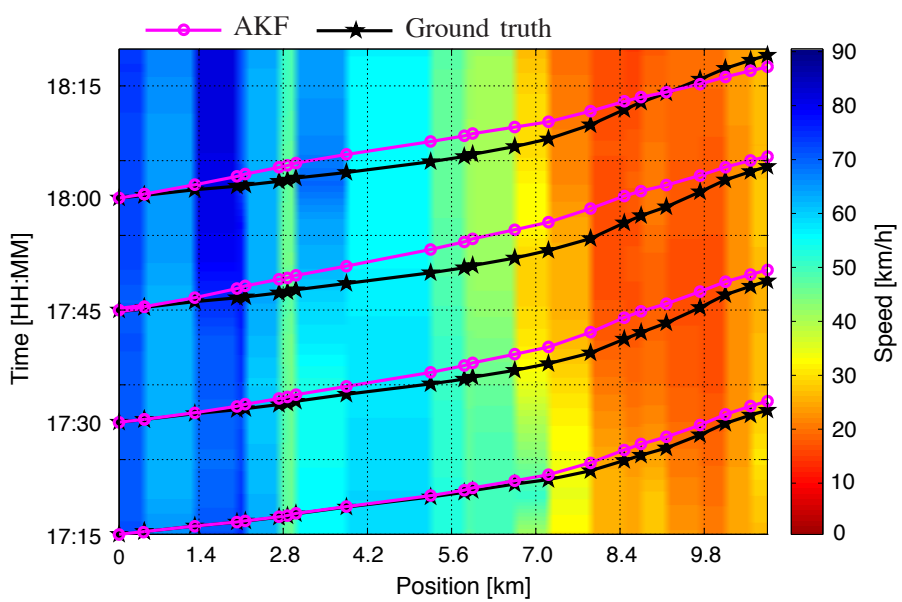

(d)

Figure 10. Performance of the travel-time forecasting algorithm. (a) Forecasting at $k_{p}=8: 45$ on September 17, 2013 (the forecasting step and horizon $H$ are 5 and 45 minutes, respectively), and (b) corresponding predicted trajectory for departure times at intervals of 15 minutes; (c) Forecasting at $k_{p}=17: 15$ on September 11,2013, and (d) corresponding predicted trajectory. The proposed method based on the adaptive Kalman filter (AKF) is shown in magenta.

time $k$, as $\widehat{\tau}_{i \rightarrow j}(k) \triangleq \sum_{\ell=i}^{j} \widehat{\tau}_{\ell}\left(k_{\ell}\right)$ where $k_{\ell}=k_{\ell-1}+\widehat{\tau}_{\ell}\left(k_{\ell-1}\right)$, and the absolute percentage error (APE) at time $k$ in the overall south ring, as $\operatorname{APE}(k) \triangleq 100\left|\tau_{1 \rightarrow M}(k)-\widehat{\tau}_{1 \rightarrow M}(k)\right| / \tau_{1 \rightarrow M}(k)$. Fig. 11 reports the cumulative distribution function (CDF) of the APE for different forecasting horizons $H$ 's using the proposed algorithm (black) and the average of historical data (red), 


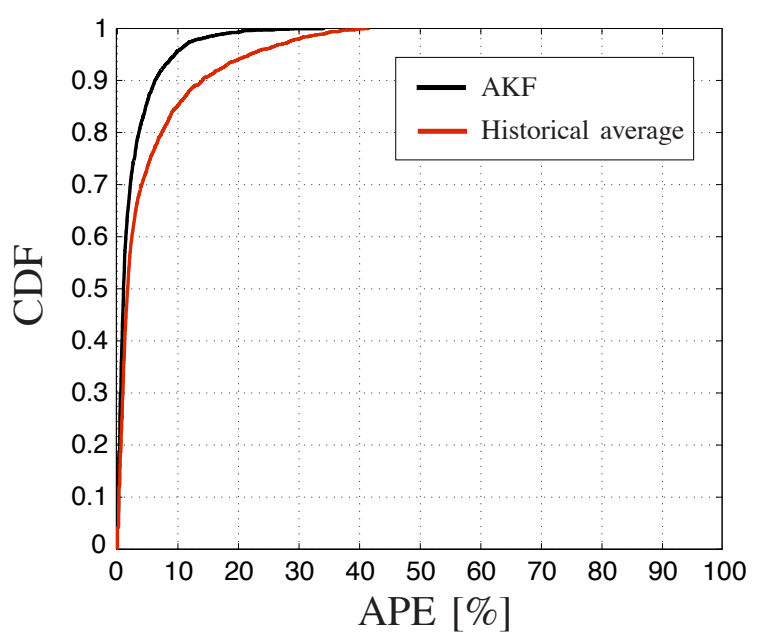

(a)

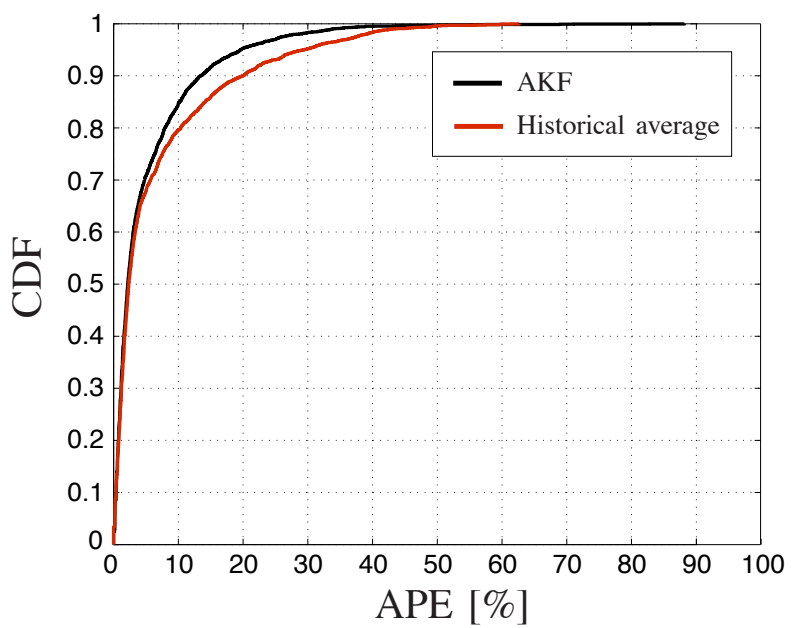

(b)

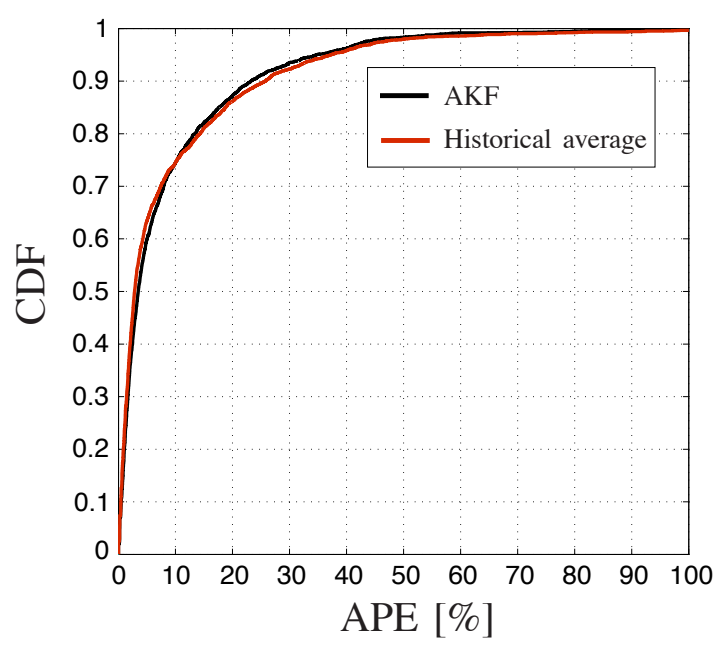

(c)

Figure 11. Performance of the travel-time forecasting algorithm. Cumulative density function $(\mathrm{CDF})$ of the absolute percentage error (APE) using the proposed algorithm (AKF, black) and a simple average of historical data (red), for different forecasting horizons: (a) $H=0$ minutes (present time); (b) $H=15$ minutes; (c) $H=45$ minutes.

when $k_{p}$ varies between 6:00 and 22:00 for 15 working days. As it is evident in Figs. 11(a)(c), the smaller $H$ the more accurate the forecasting: moreover, although the performance of the proposed algorithm is always comparable or superior than that obtained with the historical average, for large $H$, as expected, the differences between the two approaches become negligible. 


\section{Traffic-Density Estimation}

In this final section, we illustrate the performance of a recently-developed Luenbergerlike traffic density estimator based on the graph-constrained SMM: for the detailed mathematical formulation, the reader is referred to [9], [11], [23]. The south ring has been subdivided in 48 cells with average length of 220 meters and the density in each cell has been reconstructed using the model-based observer and the available flow measurements: the data have been aggregated to 1 minute and then resampled to 5 seconds, and the SMM has been automatically calibrated using a robust algorithm similar to the one proposed in [25], cf. "Level 2" above. Figs. 12(b), (d) show the density contour estimated by the proposed observer on Friday, February 28, 2014 (the eve of French student holidays, when the south ring was heavily congested all afternoon), and on Friday, March 7, 2014, respectively. Figs. 12(a), (c) report the corresponding measured densities, that is the densities reconstructed from the available flow and speed measurements in the collection points of the south ring (our ground truth). The gray vertical stripes in Fig. 12(a) correspond to three collection points (Meylan, Gabriel Péri entrance 1, SMH Centre exit) which were not operative on February 28. In spite of the missing data, we observe a satisfactorily agreement between the measured and estimated densities.

\section{Conclusions and Future Work}

In this article we have described the Grenoble traffic lab (GTL), a novel experimental platform for advanced traffic research, and we have presented some activities built around it that our group has recently carried out.

GTL is an ongoing and living research project, which serves as a basis for more ambitious forthcoming undertakings: in the near future, we aim at customizing the GTL web interface for 


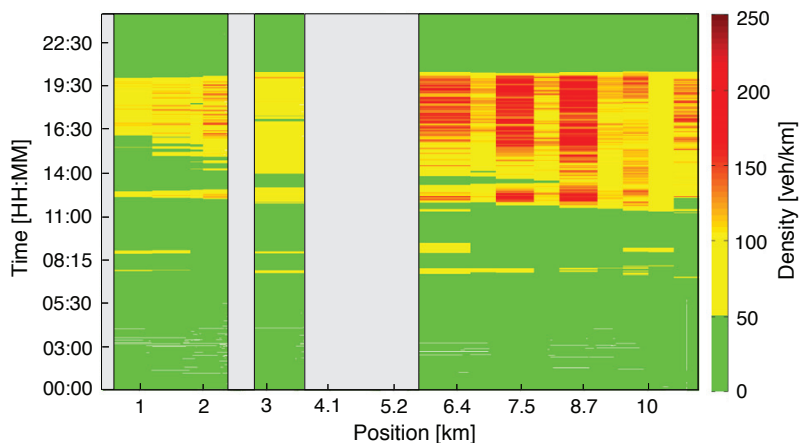

(a)

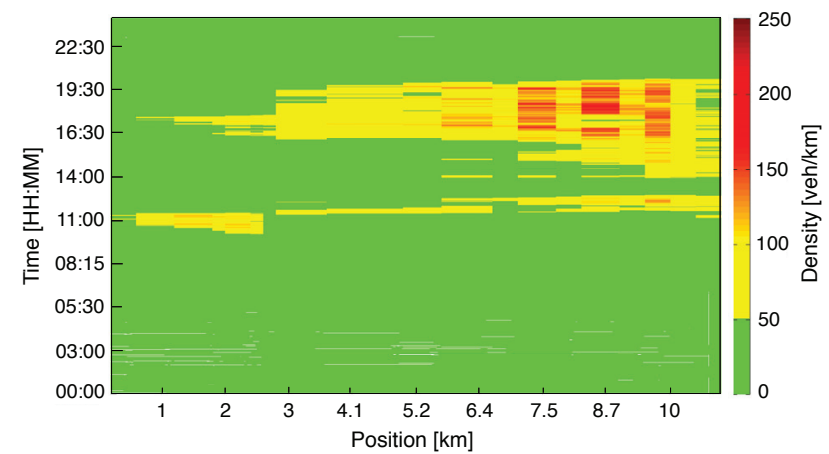

(c)

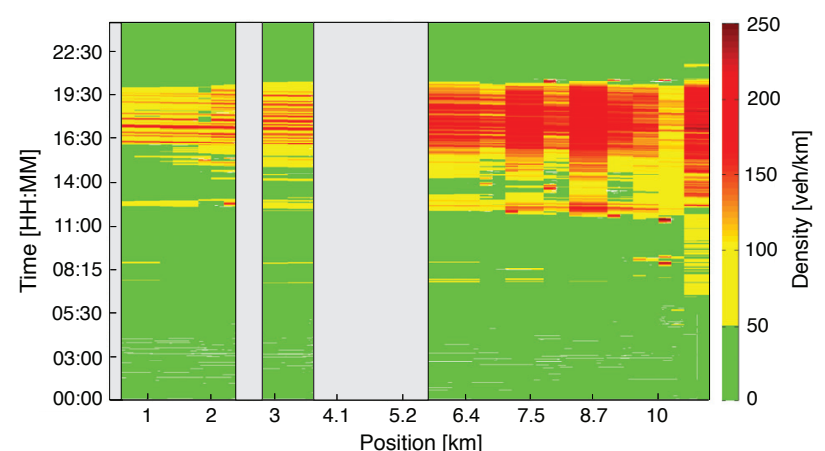

(b)

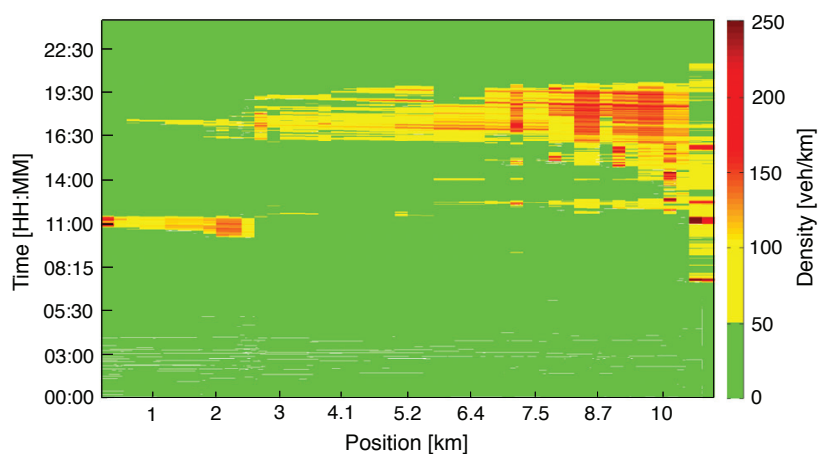

(d)

Figure 12. Density reconstruction in the south ring using the CTM-based observer. (a) Measured density, and (b) estimated density on February 28, 2014; (c) Measured density, and (d) estimated density on March 7, 2014.

different categories of vehicles, e.g. fire trucks, ambulances, police and passenger cars. Looking further into the future, we also plan to extend our network of sensors to the major urban arterials of Grenoble in order to have a wider city-level coverage of traffic behavior, and to combine traffic data coming from heterogeneous sensors (e.g., magnetometers and GPS-equipped vehicles).

\section{References}

[1] D. Schrank, T. Lomax, and B. Eisele. TTI's 2011 Urban Mobility Report. Technical report, The Texas A\&M University System. http://mobility.tamu.edu. 
[2] INRIX National Traffic Scorecard Annual Report. www.inrix.com/scorecard/.

[3] N. Geroliminis and C.F. Daganzo. Existence of urban-scale macroscopic fundamental diagrams: Some experimental findings. Transport. Res. B-Meth., 42(9):759-770, 2008.

[4] J.C. Herrera, D.B. Work, R. Herring, X.J. Ban, Q. Jacobson, and A.M. Bayen. Evaluation of traffic data obtained via GPS-enabled mobile phones: The Mobile Century field experiment. Transport. Res. C-Emer., 18(4):568-583, 2010.

[5] M. Treiber and A. Kesting. Traffic Flow Dynamics: Data, Models and Simulation. Springer, 2013.

[6] S. Blandin, A. Couque, A.M. Bayen, and D.B. Work. On sequential data assimilation for scalar macroscopic traffic flow models. Physica D, 241(17):1421-1440, 2012.

[7] C.F. Daganzo. The cell transmission model: a dynamic representation of highway traffic consistent with the hydrodynamic theory. Transport. Res. B-Meth., 4(28):269-287, 1994.

[8] L. Muñoz, X. Sun, R. Horowitz, and L. Alvarez. Traffic density estimation with the cell transmission model. In Proc. Amer. Contr. Conf., pages 3750-3755, 2003.

[9] C. Canudas de Wit, L. León Ojeda, and A.Y. Kibangou. Graph Constrained-CTM observer design for the Grenoble south ring. In Proc. 13th IFAC Symp. Contr. Transp. Syst., volume 13, pages 197-202, 2012.

[10] A.A. Kurzhanskiy and P. Varaiya. Guaranteed prediction and estimation of the state of a road network. Transport. Res. C-Emer., 21(1):163-180, 2012.

[11] F. Morbidi, L. León Ojeda, C. Canudas de Wit, and I. Bellicot. Robust mode selection for highway traffic density estimation. In Proc. Europ. Contr. Conf., pages 2576-2580, 2014.

[12] Y. Sun and D.B. Work. A Distributed Local Kalman Consensus Filter for Traffic Estimation. In Proc. 53rd IEEE Conf. Dec. Contr., pages 6484-6491, 2014.

[13] X. Sun, L. Mun̄oz, and R. Horowitz. Highway traffic state estimation using improved 
mixture Kalman filters for effective ramp metering control. In Proc. 42nd IEEE Conf. Dec. Contr., volume 6, pages 6333-6338, 2003.

[14] A. Lemarchand, J.-J. Martinez, and D. Koenig. Smooth Switching $\mathrm{H}_{\infty}$ PI Controller for Local Traffic On-ramp Metering, an LMI Approach. In Proc. 18th IFAC World Congr., pages $13882-13887,2011$.

[15] A. Ferrara, A. Nai Oleari, S. Sacone, and S. Siri. Freeways as Systems of Systems: A Distributed Model Predictive Control Scheme. IEEE Syst. J., 2014, in press.

[16] A. Chow, V. Dadok, G. Dervisoglu, G. Gomes, R. Horowitz, A.A. Kurzhanskiy, J. Kwon, X.Y. Lu, A. Muralidharan, S. Norman, R.O. Sánchez, and P. Varaiya. TOPL: Tools for operational planning of transportation networks. In Proc. ASME Dyn. Syst. Contr. Conf., pages 1035-1042, 2008.

[17] A.A. Kurzhanskiy and P. Varaiya. Active traffic management on road networks: a macroscopic approach. Philos. Trans. Roy. Soc. A, 368(1928):4607-4626, 2010.

[18] Transport Simulation Systems. AIMSUN traffic modelling software. www • aimsun • com.

[19] D. Pisarski and C. Canudas de Wit. Optimal Balancing of Freeway Traffic Density: Application to the Grenoble South Ring. In Proc. Europ. Contr. Conf., pages 4021-4026, 2013

[20] D. Bianchi, A. Ferrara, and M.D. Di Benedetto. Networked Model Predictive Traffic Control with Time Varying Optimization Horizon: The Grenoble South Ring Case Study. In Proc. Europ. Contr. Conf., pages 4039-4044, 2013.

[21] B.L. Smith, W.T. Scherer, and J.H. Conklin. Exploring Imputation Techniques for Missing Data in Transportation Management Systems. Trans. Res. Rec., 1836(1):132-142, 2003.

[22] A. Muralidharan and R. Horowitz. Imputation of Ramp Flow Data for Freeway Traffic Simulation. Trans. Res. Rec., 2099(1):58-64, 2009. 
[23] L. Léon Ojeda. Short-term multi-step ahead traffic forecasting. $\mathrm{PhD}$ thesis, Université de Grenoble, GIPSA-lab, Département d'Automatique, July 2014. http://necs.inrialpes.fr/media/documents/publications/theses/ Leon_ojeda-thesis.pdf.

[24] Grenoble Traffic Lab - Home page. http://necs.inrialpes.fr/pages/ grenoble-traffic-lab.php.

[25] G. Dervisoglu, G. Gomes, J. Kwon, R. Horowitz, and P. Varaiya. Automatic calibration of the fundamental diagram and empirical observations on capacity. In Transp. Res. Board 88th Annual Meeting, number 09-3159, 2009.

[26] L. Int Panis, S. Broekx, and R. Liu. Modelling instantaneous traffic emission and the influence of traffic speed limits. Sci. Total Environ., 371(1):270-285, 2006.

[27] J.-J. Martinez and C. Canudas de Wit. A safe longitudinal control for adaptive cruise control and stop-and-go scenarios. IEEE Trans. Control Syst. Technol., 15(2):246-258, 2007.

[28] GTL web interface. http://gtl4.inrialpes.fr/gtl/.

[29] Ministère de l'Écologie du Développement durable et de l'Énergie. Diffusion numérique de l'information routière. diffusion-numerique.info-routiere.gouv. fr.

[30] M. Su and J. Luk. Evaluation of Sensys Networks Equipment: Stage 3, Tests on Monash Freeway. Technical Report VC72270-3, ARRB Group Ltd, 2007. www •arrb.com.au/ Information-services/Publications/.

[31] Sensys Networks. Evaluation of the Sensys ${ }^{\mathrm{TM}}$ Wireless Vehicle Detection System by the California Center for Innovative Transportation. Technical report, 2007. www . sensysnetworks.com/white-papers/.

[32] Sensys Networks. Count Accuracy Assessment of the Sensys ${ }^{\mathrm{TM}}$ Wireless Vehicle Detection System by the Texas Transportation Institute. Technical report, 2007. www . 
sensysnetworks.com/white-papers/.

[33] R.T. Rockafellar. Network Flows and Monotropic Optimization. Athena Scientific, 1998.

[34] L. León Ojeda, A.Y. Kibangou, and C. Canudas de Wit. Online Dynamic Travel Time Prediction using Speed and Flow Measurements. In Proc. Europ. Contr. Conf., pages 4045-4050, 2013.

[35] W.-H. Lin. A Gaussian maximum likelihood formulation for short-term forecasting of traffic flow. In Proc. IEEE Int. Transp. Sys., pages 150-155, 2001.

[36] B.L. Smith, B.M. Williams, and R.K. Oswald. Comparison of parametric and nonparametric models for traffic flow forecasting. Transport. Res. C-Emer., 10(4):303-321, 2002.

[37] R. Chrobok, O. Kaumann, J. Wahle, and M. Schreckenberg. Different methods of traffic forecast based on real data. Eur. J. Oper. Res., 155(3):558-568, 2004.

[38] L. León Ojeda, A.Y. Kibangou, and C. Canudas de Wit. Adaptive Kalman Filtering for Multi-Step ahead Traffic Flow Prediction. In Proc. Amer. Contr. Conf., pages 4731-4736, 2013.

[39] R.F. Stengel. Optimal Control and Estimation. Courier Dover Publications, 2012.

\section{Acknowledgements}

This research has been supported by the EU FP7/2010-14 HYCON2 NoE under grant agreement no. 257462, by the project MOCoPo "Measuring and mOdelling traffic COngestion and POllution" through the PREDIT, by the "Fonds d'Intervention de l'UJF", pôle scientifique MSTIC, and by the Carnot iC LSI project "Mobile Devices for Intelligent Road Traffic Prediction". The authors would like to thank the DIR-CE, Service Régional d'Explotation et d'Ingégnerie, District de Grenoble, for their support in the design of GTL data-acquisition system, and Karrus ITS for the installation and maintenance of the sensor network. 


\section{Author Information}

- Carlos Canudas de Wit received the B.Sc. degree in Electronics and Communications from the Technological Institute of Monterrey, Mexico in 1980. In 1984 he received his M.Sc. from the Department of Automatic Control of the University of Grenoble, France. In 1987 he received his Ph.D. in Automatic Control from the Polytechnic of Grenoble, France. Since then he has been working at the same department as "Directeur de recherche" at the CNRS, where he teaches and conducts research in the area of nonlinear control of mechanical systems and control over networks. He is the current leader of the joint GIPSAlab/Inria NeCS team on networked control systems. His research publications include 185 international conference papers, 63 published papers in international journals, 5 books, 9 book chapters, and 11 patents.

- Fabio Morbidi received the M.Sc. degree in Computer Engineering and Ph.D. degree in Robotics and Automation from the University of Siena, Italy, in 2005 and 2009, respectively. He was a Visiting Scholar at the University of California, Santa Barbara, USA, in 2007-2008. He held postdoctoral positions at the Northwestern University, USA, University of Texas at Arlington, USA and Inria Grenoble Rhône-Alpes, France. He is currently an Associate Professor at the University of Picardie Jules Vernes, Amiens, France. His research interests include multi-agent systems, distributed control, robot vision and road traffic estimation.

- Luis Léon Ojeda received the B.Sc. degree in Electrical Engineering from the Central University of Venezuela in 2006, the M.Sc. degree in Systems Engineering from the University La Sapienza, Italy, in 2008, and the Ph.D. in Automatic Control from the University of Grenoble, France, in July 2014. He is currently a research engineer at the IFP Energies Nouvelles, Paris. His research interests are in the area of intelligent transportation systems, specifically, traffic estimation, forecasting, and data treatment. 
- Alain Y. Kibangou has been Associate Professor at the University Grenoble Alpes, France, since 2009. He received the Ph.D. degree in control, signal, and image processing from the University of Nice Sophia Antipolis in 2005. Then, he was a Postdoctoral Researcher successively with the I3S Laboratory, Sophia Antipolis, the LAAS, Toulouse, and the GIPSA-lab, Grenoble. His research interests include distributed estimation, structural network analysis, multiway array data analysis, signal processing for communications, traffic prediction, system identification, nonlinear filtering, and consensus problems.

- Iker Bellicot was a research engineer of the NeCS team at Inria Grenoble Rhône-Alpes, France, between 2011 and 2014. He received the B.Sc. and M.Sc. both in Computer Engineering from the University Joseph Fourier, Grenoble, in 2004 and 2006, respectively.

- Pascal Bellemain is a Software Development Engineer at the Department of Automatic Control of GIPSA-lab, CNRS, Grenoble, France.

Corresponding author: Fabio Morbidi, MIS laboratory, University of Picardie Jules Verne, 33 Rue Saint-Leu, 80039 Amiens, France. Tel. +33 (0)3 $22 \quad 85 \quad 59$ 02, Email: fabio.morbidi@u-picardie.fr

\section{Sidebar 1: Fluid-Dynamic Macroscopic Models for Highway Traffic}

Macroscopic traffic models describe the evolution of vehicle positions in a highway in terms of macroscopic variables such as the density $\rho(t, x)$ and average speed $v(t, x)$ of the vehicles, where $t$ and $x$ are the time and space indices, respectively. The simplest macroscopic model is the scalar one proposed independently by Lighthill and Whitham in 1955 and by Richards in 1956 (the LWR model). This first-order model is based on the conservation law of vehicles and is described by $\frac{\partial \rho}{\partial t}+\frac{\partial \Phi(\rho, v)}{\partial x}=0$ where $\rho(t, x) \in\left[0, \rho_{m}\right]$ being $\rho_{m}$ the maximal density of cars on the highway, and the flux $\Phi(\rho, v)$ is given by $\rho v$. In most cases we can assume that the average speed $v$ depends only on the density of the vehicles (in fact, vehicles tend to 
travel at an equilibrium speed), thus $\Phi(\rho, v)=\Phi(\rho)$ and its graph is called the fundamental diagram. For simplicity, it is typically assumed that $\Phi(\rho)$ is concave and has a unique maximum in $\left(0, \rho_{m}\right)$ [S1]. In a triangular fundamental diagram, one of the most used in the literature, there are only two distinct propagation velocities of density variations, one for free traffic, $v$, and one for congested traffic, $w$. The transition from a regime to the other is determined by the critical density $\rho_{c}$. The most common integration method for the LWR model is the Godunov scheme [S2]. The discrete version of the LWR model with triangular fundamental diagram, is formulated as an iterated coupled map with time and space discretized into time steps and cells, respectively, and supplemented by a special "supply-demand" update rule to describe interactions between adjacent highway cells as well as shockwaves. This model is known as cell transmission model (CTM) [7].

\section{References}

[S1] M. Garavello, B. Piccoli, Traffic Flow on Networks: Conservation Laws Models, AIMS Series on Applied Mathematics, 2006.

[S2] R.J. LeVeque, Finite Volume Methods for Hyperbolic Problems, Cambridge University Press, 2004.

\section{Sidebar 2: How Do Magnetic Sensors Detect a Passing Vehicle?}

Magnetic sensors are passive devices that indicate the presence of a metallic object by detecting the perturbation (known as a magnetic anomaly) in the Earth's magnetic field created by the object [S3]. Fig. S1 shows the distortion induced in the Earth's magnetic field as a vehicle enters and passes through the detection zone of a magnetic sensor embedded in the roadway. In particular, Fig. S1(right) depicts the magnetic field as the vehicle approaches the 
sensor (gray rectangle). Fig. S1(center) shows the field lines of flux (red) as the vehicle begins to pass through the sensor's detection zone, and Fig. S1(left) illustrates the lines of flux when the entire vehicle is over the sensor. Two- and three-axis fluxgate magnetometers detect changes in the vertical and horizontal components of the Earth's magnetic field produced by a ferrous metal vehicle and are able to identify stopped and moving cars. Two-axis fluxgate magnetometers contain a primary winding and two secondary "sense" windings on a bobbin surrounding a high permeability soft magnetic material core. In response to the magnetic field anomaly, i.e. the magnetic signature of a vehicle, the magnetometer's electronics circuitry measures the output voltage generated by the secondary windings. The vehicle detection criterion is for the voltage to exceed a predefined threshold. Sensys Networks VDS240s are three-axis magneto-resistive sensors that measure the $x-, y$-, and $z$-components of the Earth's magnetic field. They are installed by boring a $10-\mathrm{cm}$ diameter hole approximately $6.5 \mathrm{~cm}$ deep, inserting the sensor into the hole so that it is properly aligned with the direction of traffic flow, and sealing the hole with fast drying epoxy. The sensor maintains two-way wireless communication with an access point device over a range of 23 to 46 meters. Since fluxgate magnetometers are passive devices, they do not transmit an energy field, and a portion of the vehicle must pass over the sensor for it to be detected. Therefore, a magnetometer can detect two vehicles separated by a distance of $30 \mathrm{~cm}$. This potentially makes the magnetometer as accurate as or better than an inductive loop detector at counting vehicles. However, magnetometers are not precise at locating the perimeter of a
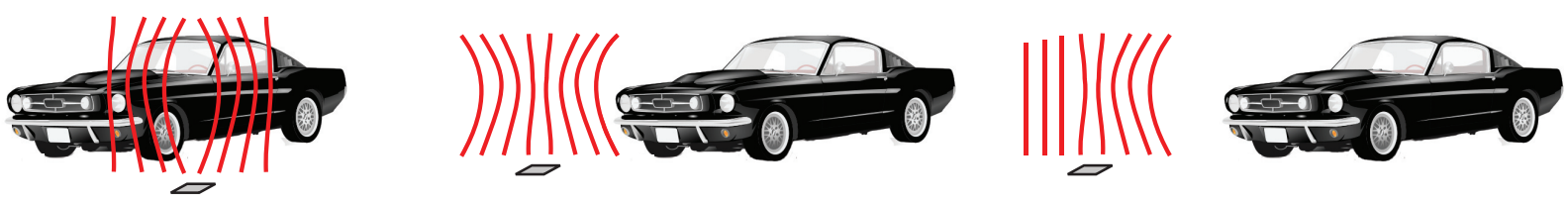

Figure S1. From right to left: perturbation of Earth's magnetic field (red lines) produced by a vehicle approaching and passing through the detection zone of a magnetic sensor (gray rectangle). 
vehicle: in fact, an uncertainty of about $45 \mathrm{~cm}$ is typically experienced. A single magnetometer is therefore seldom used for determining occupancy and speed in traffic management applications, and two closely-spaced sensors are usually preferred for that function.

\section{References}

[S3] U.S. Department of Transportation, Federal Highway Administration, Policy Information, Highway Finance Data \& Information: Chapter 4 - In-roadway Sensor Technologies, 2007, www.fhwa.dot.gov/policyinformation/index.cfm

\section{Sidebar 3: Travel Time Forecasting}

Consider a vehicle traversing the road segment $\left[x_{p}, x_{0}\right] \subset \mathbb{R}$ in the time interval $\left[t_{p}, t_{0}\right]$, see Fig. S2. We are here interested in determining a formula for the exit time $t_{0}$ (the current time) given the entry time $t_{p}$ and the extrema of the segment. Note that if the velocity field $v(t, x)$ in $\left[x_{p}, x_{0}\right]$ is known, then the infinitesimal travel time of the vehicle is given by $d t=v(t, x) d x$. By writing this equation in integral form, we obtain the following expression for $t_{0}$

$$
t_{0}=t_{p}+\int_{x_{p}}^{x_{0}} v(t, x) d x
$$

Since the velocity field $v(t, x)$ is not known in general, but suitable measurement points are available within the road segment, we can approximate $v(t, x)$ by discretizing the interval $\left[x_{p}, x_{0}\right]$. By subdividing $\left[x_{p}, x_{0}\right]$ into $n$ rectangles of width $\Delta x_{i}$ and assuming that the speed is constant in each rectangle (see Fig. S2), we can rewrite equation (S1) as

$$
t_{0}=t_{p}+\sum_{i=1}^{n} \frac{\Delta x_{i}}{v_{i}\left(\eta\left(\Delta x_{i}\right)\right)}
$$

where $v_{i}\left(\eta\left(\Delta x_{i}\right)\right)$ is the space-mean speed in the $i$-th rectangle and

$$
\eta\left(\Delta x_{i}\right)=t_{p}+\sum_{j=1}^{i-1} \frac{\Delta x_{j}}{v_{j}\left(\eta\left(\Delta x_{j}\right)\right)}
$$




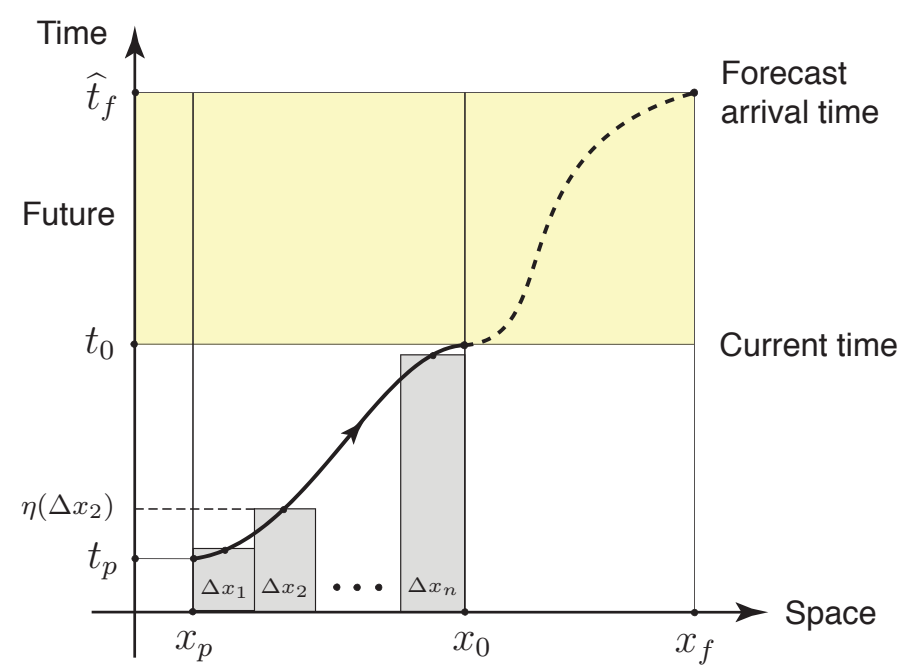

Figure S2. Computation of forecast progressive travel time.

is the time at which the vehicle reaches the upstream boundary of rectangle $i$. Note that (S3) accounts for the traffic progression along the road. Equations (S2) and (S3) define the progressive travel time (PTT). This differs from the instantaneous travel time (ITT), frequently encountered in the literature, which assumes that the conditions in each rectangle remain the same as at the entry time $t_{p}$, i.e. $t_{0}=t_{p}+\sum_{i=1}^{n} \frac{\Delta x_{i}}{v_{i}\left(t_{p}\right)}$ (in other words, time is "frozen"). Note that while the PTT is consistent with the traffic conditions that a driver encounters along the road, the computation of ITT is based on an assumption that is not necessarily verified in the real world, and which becomes more critical as the width of the rectangles $\Delta x_{i}$ increases. Let us now determine the forecast arrival time $\widehat{t}_{f}$ of the vehicle at the point $x_{f}$, given the current time $t_{0}$ and current position $x_{0}$ (see Fig. S2). By using (S2) and (S3), and assuming a space discretization in $M$ rectangles, we obtain $\widehat{t}_{f}=t_{0}+\sum_{i=1}^{M} \frac{\Delta x_{i}}{\widehat{v}_{i}\left(\widehat{\eta}\left(\Delta x_{i}\right)\right)}, \widehat{\eta}\left(\Delta x_{i}\right)=t_{0}+\sum_{j=1}^{i-1} \frac{\Delta x_{j}}{\hat{v}_{j}\left(\widehat{\eta}\left(\Delta x_{j}\right)\right)}$. By defining $\widehat{\tau}_{i}(k)$ as the forecast PTT in rectangle $i$ at the discrete time $k$, and $\widehat{\tau}_{x_{0} \rightarrow i}(k)$ as the cumulative PTT from the entry point $x_{0}$ to the downstream boundary of rectangle $i$ at the discrete time $k$, we find $\widehat{\tau}_{x_{0} \rightarrow i}(k)=\widehat{\tau}_{x_{0} \rightarrow i-1}(k)+\widehat{\tau}_{i}\left(k+\widehat{\tau}_{x_{0} \rightarrow i-1}(k)\right), \widehat{\tau}_{i}(k)=\frac{\Delta x_{i}}{\hat{v}_{i}\left(\widehat{\tau}_{x_{0} \rightarrow i-1}(k)\right)}$ where $i \in\{1, \ldots, M\}$, $k \in\left\{k_{0}+1, \ldots, k_{0}+H\right\}$ and $H$ is the forecasting horizon. 